\title{
Phytotoxin production in Aspergillus terreus is regulated by independent environmental signals
}

\section{Markus Gressler ${ }^{1}$, Florian Meyer ${ }^{2}$, Daniel Heine ${ }^{2}$, Peter Hortschansky ${ }^{3}$, Christian Hertweck ${ }^{2}$, Matthias Brock ${ }^{4,5 * \dagger}$}

${ }^{1}$ Microbial Biochemistry and Physiology, Leibniz Institute for Natural Product Research and Infection Biology, Hans Knoell Institute, Jena, Germany; ${ }^{2}$ Biomolecular Chemistry, Leibniz Institute for Natural Product Research and Infection Biology, Hans Knoell Institute, Jena, Germany; ${ }^{3}$ Molecular and Applied Microbiology, Leibniz Institute for Natural Product Research and Infection Biology, Hans Knoell Institute, Jena, Germany; ${ }^{4}$ Institute for Microbiology, Friedrich Schiller University, Jena, Germany; ${ }^{5}$ Fungal Genetics and Biology Group, School of Life Sciences, University of Nottingham, Nottingham, United Kingdom

Abstract Secondary metabolites have a great potential as pharmaceuticals, but there are only a few examples where regulation of gene cluster expression has been correlated with ecological and physiological relevance for the producer. Here, signals, mediators, and biological effects of terrein production were studied in the fungus Aspergillus terreus to elucidate the contribution of terrein to ecological competition. Terrein causes fruit surface lesions and inhibits plant seed germination.

Additionally, terrein is moderately antifungal and reduces ferric iron, thereby supporting growth of A. terreus under iron starvation. In accordance, the lack of nitrogen or iron or elevated methionine levels induced terrein production and was dependent on either the nitrogen response regulators AreA and AtfA or the iron response regulator HapX. Independent signal transduction allows complex sensing of the environment and, combined with its broad spectrum of biological activities, terrein provides a prominent example of adapted secondary metabolite production in response to environmental competition.

DOI: 10.7554/eLife.07861.001

\section{Introduction}

Fungal secondary metabolites (SMs) are often encoded by gene clusters and have been the subject of intensive analysis in recent years. This has resulted from the large number of completed fungal genomes as well as the intrinsic interest of SMs as environmental/pathogenic agents and their potential for pharmacological use. However, there are few examples where the regulation of the expression of these clusters has been shown to be ecologically/physiologically relevant (Rohlfs and Churchill, 2011). Except for penicillin and perhaps aflatoxin and gliotoxin, the potential real-life functions of most SMs are unknown (Bhatnagar et al., 2006; Vargas et al., 2014). Most clusters contain a pathway-specific transcription factor, and the common approach is to artificially over-express the cluster genes by constitutive expression of this transcription factor and chemically characterise the product (Yin and Keller, 2011). In most cases the relevance of the SM is not known.

In this study the biological function of terrein from the filamentous ascomycete Aspergillus terreus was investigated. A. terreus is a filamentous ascomycete of biotechnological and medical importance since it produces itaconic acid (Steiger et al., 2013) and lovastatin (Bizukojc and Ledakowicz, 2015), 
eLife digest Organisms produce a wide variety of small molecules called metabolites through the break down of food and other chemical reactions. Some of these molecules-known as primary metabolites-are required for growth, reproduction and other vital processes. Other molecules called secondary metabolites are not strictly required by the organism, but generally have other roles that may improve the individual's ability to survive and reproduce.

Fungi and other microbes produce a large variety of secondary metabolites, many of which are used as medicines to treat diseases in humans and other animals. For example, a molecule called lovastatin-which is produced by a fungus known as Aspergillus terreus-can reduce a human patient's risk of heart disease. However, it is not known what role many secondary metabolites play in the microbe that produced them.

A. terreus lives in the soil, but it can also infect plants and animals. In addition to lovastatin, it also makes another secondary metabolite called terrein. A recent study identified the genes responsible for making terrein, and discovered that this molecule is harmful to plant cells and may help the fungus to colonize and thrive in the area immediately around plant roots, which is known as the rhizosphere.

Here, Gressler et al. studied how terrein may help the fungus to cope with competitors in this environment. The experiments show that terrein increases the availability of iron and inhibits the growth of competing microbes. A shortage of iron or nitrogen-containing nutrients can stimulate the fungus to produce terrein, and elevated levels of a molecule called methionine have the same effect. These conditions are commonly found in the rhizosphere and further experiments identified several proteins in the fungus that are required for sensing them.

Gressler et al.'s findings suggest that terrein helps to ensure that the fungus has sufficient nitrogen and iron to thrive in the rhizosphere. Also, this study confirms that the production of secondary metabolites in microbes can happen in response to elaborate cues from the environment, which may explain why only a limited number of secondary metabolites are produced by microbes when they are grown in the laboratory. Future studies will analyze other ways to activate the production of secondary metabolites outside of the microbe's normal environment, which may lead to the discovery of new important drugs.

DOI: 10.7554/eLife.07861.002

but it is also a causative agent of life-threatening invasive aspergillosis in immunocompromised patients (Baddley et al., 2003; Slesiona et al., 2012b) and has recently been described as a pathogen of potato leaves (Louis et al., 2013).

While searching for a candidate protein required for pigment synthesis in $A$. terreus conidia, we serendipitously identified the gene cluster responsible for terrein production (Zaehle et alo, 2014), which is the major SM formed by $A$. terreus. Terrein production is highly pronounced on sugar-rich plant-derived media such as potato dextrose broth (PDB) (Zaehle et al., 2014) and shows phytotoxic activities such as inhibition of seed germination and lesion formation on fruit surfaces (Kamata et al., 1983; Zaehle et al., 2014).

The terrein biosynthesis gene cluster consists of 11 genes (terA-J, terR), whereby terA encodes the key enzyme, which is a non-reducing polyketide synthase (Zaehle et al., 2014), and terR codes for the transcriptional activator TerR containing a GAL4-type $\mathrm{Zn}_{2} \mathrm{Cys}_{6}$ binuclear cluster DNA-binding domain (Gressler et al., 2015a), as frequently found in transcriptional activators of fungal SM gene clusters. Although activation of cluster genes is strictly TerR-dependent, no signals that lead to TerR activation under in vivo conditions have yet been identified.

Since terrein is the major SM produced by A. terreus-gram scales are easily achieved-we assumed a benefit from its production in the natural habitat. To elucidate this question, a detailed knowledge on the inducing factors stimulating terrein production and analyses of its biological activities were required. Therefore, we aimed to investigate environmental signals that result in ter $R$ expression and, eventually, the production of terrein. From these analyses, the impact of different global transcription factors on cluster induction was deduced. Two global transcription factors sense the quality and availability of nitrogen sources and specifically respond to the plant and fruit environment. Additionally, the iron responsive regulon plays a vital role in cluster induction, which indicates a specific contribution of terrein in modulating iron homeostasis. 


\section{Results}

\section{Fruits induce the production of terrein}

In previous studies we showed that terrein is produced on plant-derived media such as PDB, which is in agreement with its phytotoxic biological activity (Zaehle et al., 2014). To address the question of specific conditions that induce the gene cluster, an $A$. terreus reporter strain was generated expressing the $\beta$-galactosidase gene lac $Z$ from Escherichia coli under control of the terrein synthase promoter PterA (PterA:lacZ). Due to the dependence of PterA expression on TerR, this strain served as a direct indicator of terR expression and terrein production.

In agreement with a lack of terrein production, $\beta$-galactosidase activity was near the detection limit when the A. terreus PterA:lacZ strain was grown on glucose minimal medium. In contrast, and in agreement with previous observations, a 200-500 fold induction was observed on PDB medium (Figure 1A). Similarly, on Sabouraud and yeast extract-peptone-dextrose (YPD) medium, induction levels reached $10-30 \%$ compared with PDB. However, potato broth or casamino acids did not induce the cluster without the addition of glucose, indicating that glucose appears to be required for terrein production rather than repressing gene cluster induction as shown for other SM gene clusters (Theilgaard et al., 1997; Brakhage et al., 2004; Gressler et al., 2011). Indeed, when glucose medium was supplemented with $1 \%$ casamino acids as the nitrogen source, a 20-30 fold activation was detected. Since terrein can cause lesions on fruit surfaces and inhibits plant seed germination (Kamata et al., 1983; Zaehle et al., 2014), we assumed that sugar-rich fruit and root juices might have a strong stimulatory capacity. Therefore, we cultivated the reporter strain on banana, carrot, peach, and apple juice. $\beta$-Galactosidase activities from these media exceeded the activity of the already strong inducing PDB medium (Figure 1B) by a factor of at least five. Additionally, in subsequent LC analyses of culture extracts, a distinct ultraviolet signal for terrein was detected (Figure 1-figure supplement 1A-D). This led us to infect fresh bananas with the A. terreus $\triangle a k u B$ strain (the parental strain for gene deletions; Gressler et al., 2011), a $\Delta$ terR mutant lacking the transcriptional activator, and a $\Delta$ terA deletion mutant that lacks the key polyketide synthase from the cluster.

Ethyl acetate extraction of bananas infected with $\Delta a k u B$ strain revealed high amounts of terrein, whereas no terrein was detected after infection with the $\Delta$ terR or $\Delta$ terA mutant (Figure 1C). Similar results were obtained when fresh apples or nectarines were infected with the three $A$. terreus strains (Figure 1-figure supplement 1E,F). Furthermore, the extracts of bananas infected with $A$. terreus wild-type (and $\triangle a k u B$ ) caused strong lesion formation on fresh banana peels, which was hardly observed with extracts of bananas infected with the mutants (Figure 1D). Taken together, these results confirm terrein production in a natural habitat of fruit infection, although the specific factors leading to terrein production still remain unclear.

\section{Methionine supplementation acts as an inducing signal in non-inducing media}

Since casamino acid-supplemented glucose medium revealed moderate gene cluster activation (Figure 1A), we assumed that specific amino acids could act as inducers. Therefore, we tested the inducing effect of amino acids as sole nitrogen sources by preparing six distinct pools that covered all 20 canonical proteinogenic amino acids (Figure 2-figure supplement 1).

While most of the pools did not activate terA expression, the pool with aliphatic amino acids caused moderate induction and media containing the sulphur-rich amino acids cysteine and methionine strongly induced gene expression, especially after $72 \mathrm{hr}$ of cultivation (Figure 2-figure supplement 1). In the presence of the preferred nitrogen source glutamine, the induction by aliphatic amino acids was mainly lost. Also, the activation by cysteine was low and strongly concentration-dependent (Figure 2A). In contrast, methionine provoked a significant induction even at low concentrations. Therefore, we also analysed the effect of other sulphur-containing compounds in two concentrations (Figure 2B). However, no induction was observed when homocysteine, cystathionine, glutathione, dimethylsulfoxide, sulfate, or sulfide were tested. This indicates that methionine itself rather than a sulphur source causes the induction. 


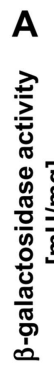

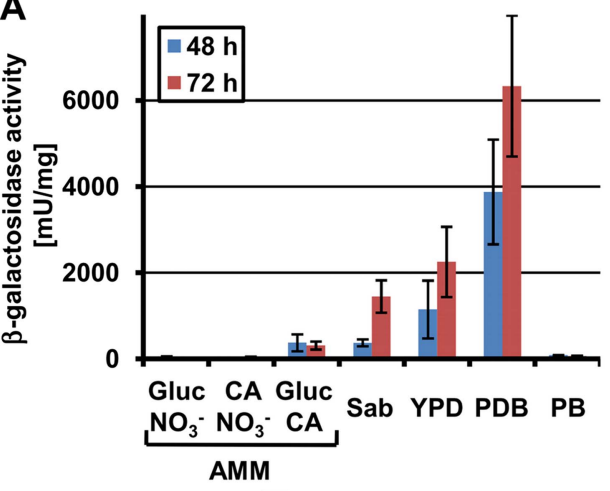

C

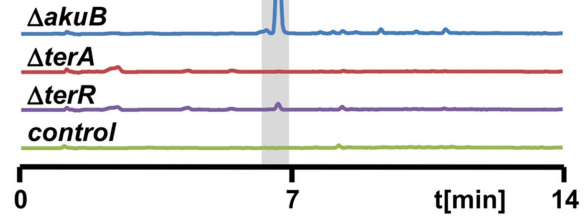

B

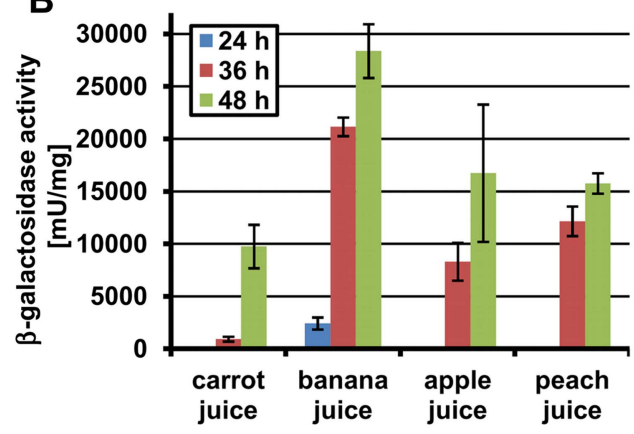

D

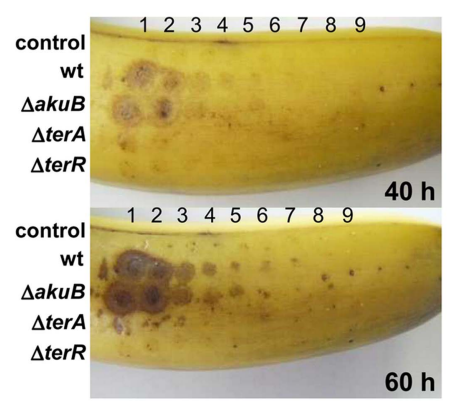

Figure 1. Terrein production and expression of terA on plant-derived media. (A) Promoter activity of strain PterA:lacZ after $48 \mathrm{hr}$ and $72 \mathrm{hr}$ on minimal media supplemented with glucose (Gluc), casamino acids (CA), or glucose + casamino acids (Gluc CA) or the complex media Sabouraud (Sab), yeast extract-peptone-dextrose (YPD), potato dextrose broth (PDB) or potato extract (PB). (B) Promoter activity of strain PterA:lacZ grown for 24, 36 and $48 \mathrm{hr}$ in carrot, banana, apple, and peach juice. (C) High performance liquid chromatography analysis of banana extracts infected with Aspergillus terreus SBUG844 strains $\Delta a k u B, \Delta a k u B \Delta t e r A$ or $\Delta a k u B \Delta t e r R$. A mockinfected fruit served as control. 1-terrein. (D) Lesion formation on banana surfaces caused by extracts shown in (C). Photographs were taken after 40 and $60 \mathrm{hr}$. Lesions only occur with extracts from the wild-type and the parental strain of the mutants $(\Delta a k u B)$. Numbers indicate the serial twofold dilution of the extracts starting from undiluted crude extracts down to 1:256 dilutions.

DOI: 10.7554/eLife.07861.003

The following figure supplements are available for figure 1:

Figure supplement 1. High performance liquid chromatography (HPLC) analysis of extracts from Aspergillus terreus strains after cultivation in fruit juices and from infected nectarines and apples.

DOI: 10.7554/eLife.07861.004

Figure supplement 2. $\beta$-Galactosidase activity of PterA:lacZ grown in banana juice without (control) or with different supplementations.

DOI: 10.7554/eLife.07861.005

\section{Nitrogen starvation acts as a second inducing signal and promotes terrein production during fruit infection}

Methionine and PDB medium induced the terrein biosynthesis gene cluster to a similar extent, but remained approximately five times below that of banana juice. This suggested that additional or alternative inducing signals might exist. Fruit juices are rich in sugars and the $\mathrm{C}: \mathrm{N}$ ratio in fruits is very high, which could result in severe nitrogen limitation at a later growth state. Therefore, another set of experiments was performed in which the total concentration of amino acids was either high $(50 \mathrm{mM})$ or low $(10 \mathrm{mM})$ and PterA induction from the lacZ reporter strain was determined at different time points (Figure 3A). While high concentrations of amino acids or inorganic nitrogen sources such as nitrate or ammonia did not induce the cluster, low nitrogen contents resulted in a time-dependent 40-400 fold induction. To correlate gene expression with nitrogen exhaustion, the nitrogen consumption from a medium supplemented with $10 \mathrm{mM}$ ammonium chloride was monitored and cluster induction was 


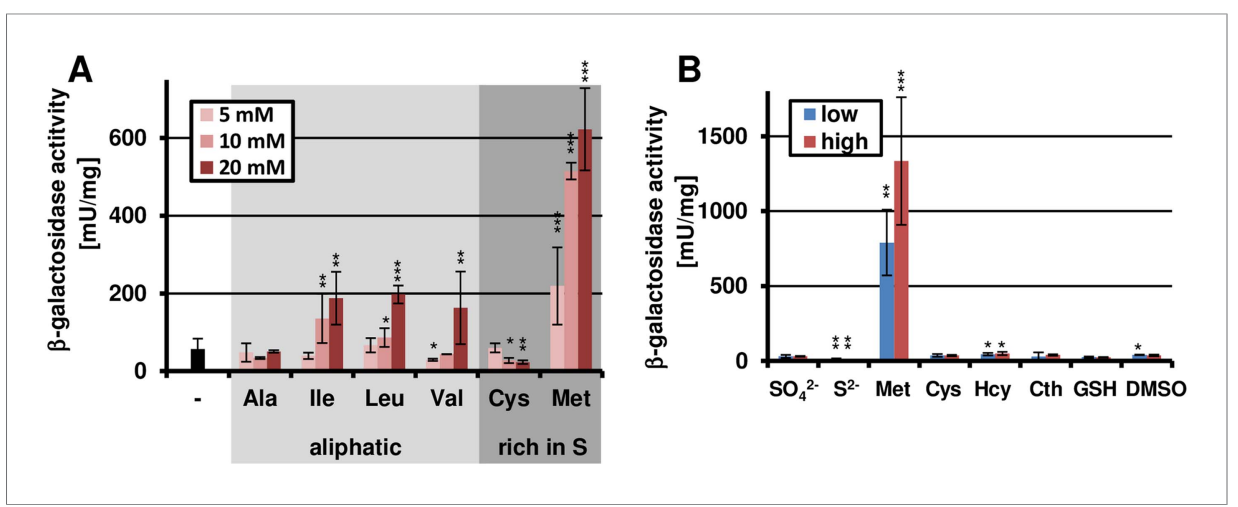

Figure 2. Methionine-dependent terA expression. (A) $\beta$-Galactosidase activity of SBUG844_PterA:lacZ after $48 \mathrm{hr}$ of cultivation in glutamine-containing minimal media in combination with 5, 10, or $20 \mathrm{mM}$ of the aliphatic (Ala, lle, Leu, Val) or sulphur-containing amino acid (Cys, Met). Significance calculated against the glutamine control. (B) $\beta$-Galactosidase assay of SBUG844_PterA:lacZ in the presence of various sulphur sources. Glutamines containing minimal media were supplemented with low $(5 \mathrm{mM})$ or high $(10 \mathrm{mM})$ concentrations of $\mathrm{Na}_{2} \mathrm{SO}_{4}, \mathrm{Na}_{2} \mathrm{~S}$, methionine (Met), cysteine (Cys), homocysteine (Hcy), reduced gluthathione (GSH), and dimethylsulfoxide (DMSO). Cystathionine (Cth) was used in final concentrations of 1 and $3 \mathrm{mM}$. Activity was determined after $48 \mathrm{hr}$ of growth. Significance calculated against medium supplemented with sodium sulfate. All cultivations were performed in biological triplicates and activity determinations were made in technical duplicates. Statistical significance was calculated by the Student's paired t-test with a two-tailed distribution: ${ }^{*} p<0.05,{ }^{* \star} p<0.01 ;{ }^{* \star *} p<0.001$

DOI: $10.7554 /$ LLife. 07861.006

The following figure supplement is available for figure 2 :

Figure supplement 1. Amino acid-dependent terA expression.

DOI: 10.7554/eLife.07861.007

simultaneously analysed. Indeed, as soon as nitrogen levels reached the detection limit, cluster expression was strongly induced (Figure 3B). Subsequently, a culture shift experiment was performed in which mycelium was pre-grown in the presence of $70 \mathrm{mM}$ ammonium chloride and shifted to medium either with or without a nitrogen source (Figure 3C). While no reporter activity and terrein formation was observed from cultures of the nitrogen-rich medium, high $\beta$-galactosidase activity accompanied by terrein accumulation was observed in the nitrogen starvation medium.

These results confirmed that nitrogen starvation acts as an inducing factor that might be responsible for the high terrein production levels on fruit juices. In agreement, when ammonium chloride was added to banana juice, $\beta$-galactosidase activity was strongly reduced (Figure 1-figure supplement 2). Similarly, the PterA:lacZ reporter strain displayed high $\beta$-galactosidase activity when bananas were directly infected (Figure 3E). When bananas were supplemented with ammonium chloride prior to infection with the wild-type, ethyl acetate extracts of the bananas revealed a $50 \%$ reduction in terrein content compared with bananas without nitrogen supplementation (Figure 3F). This finding clearly indicates that nitrogen limitation is a major inducer for terrein production under natural conditions. However, it should be mentioned that the presence of sugars was always required, since especially hexoses from mono- and disaccharides provoked strong expression under nitrogen-limited conditions (Figure 3D), which is in direct contrast to the production of dihydroisoflavipucine in $A$. terreus that belongs to the class of fruit and root rot toxins. This metabolite is only produced in the strict absence of sugars and requires preferred nitrogen sources such as glutamine or asparagine for induction (Gressler et al., 2011).

\section{AreA is the main global nitrogen regulator in $A$. terreus}

Nitrogen starvation and methionine marked important signals for terrein cluster induction. To unveil the global regulators that could be involved in signal transduction, the $A$. terreus genome was analysed for the presence of transcription factors known to play a role in nitrogen sensing, cross pathway control of amino acid synthesis, and stress response. The global nitrogen regulator AreA (ATEG_07264) (Arst and Cove, 1973; Hynes, 1975; Davis et al., 2005), the cross pathway control 
A

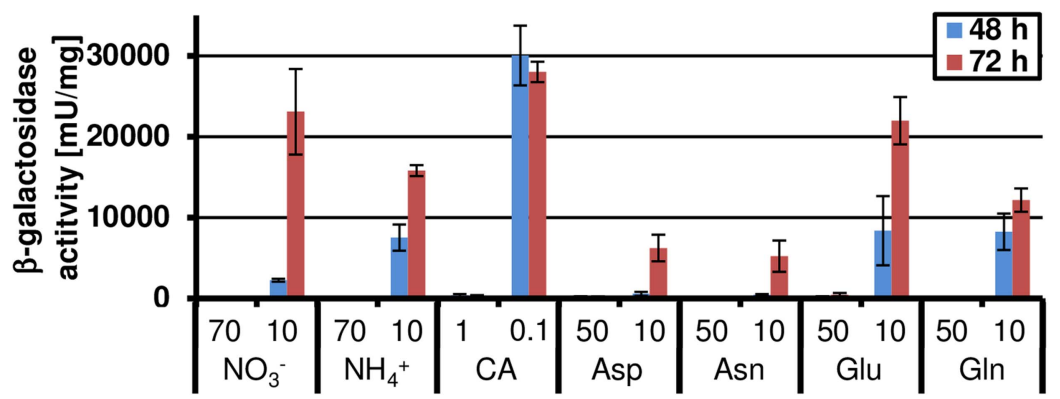

B

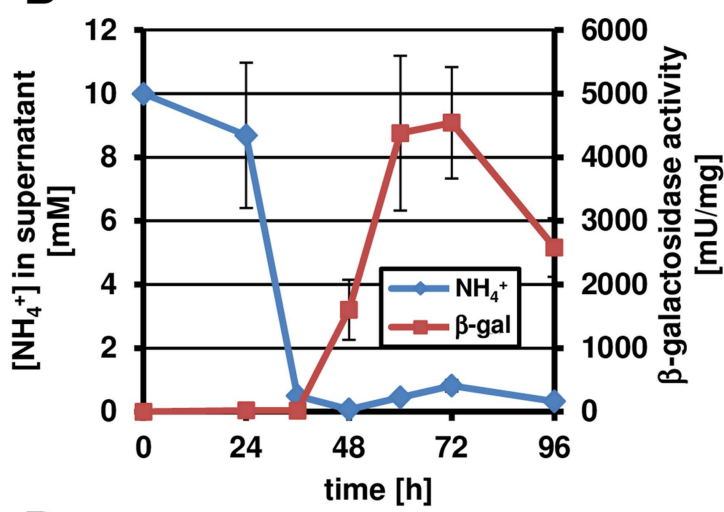

C

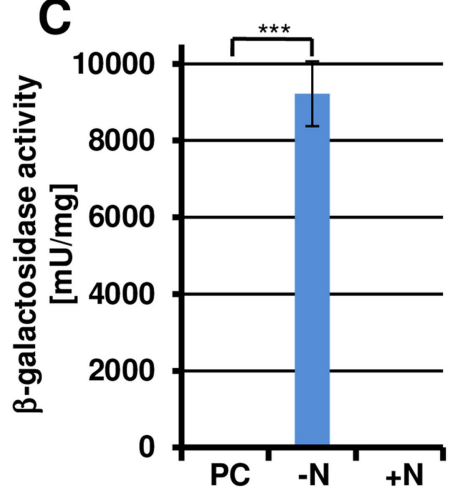

D
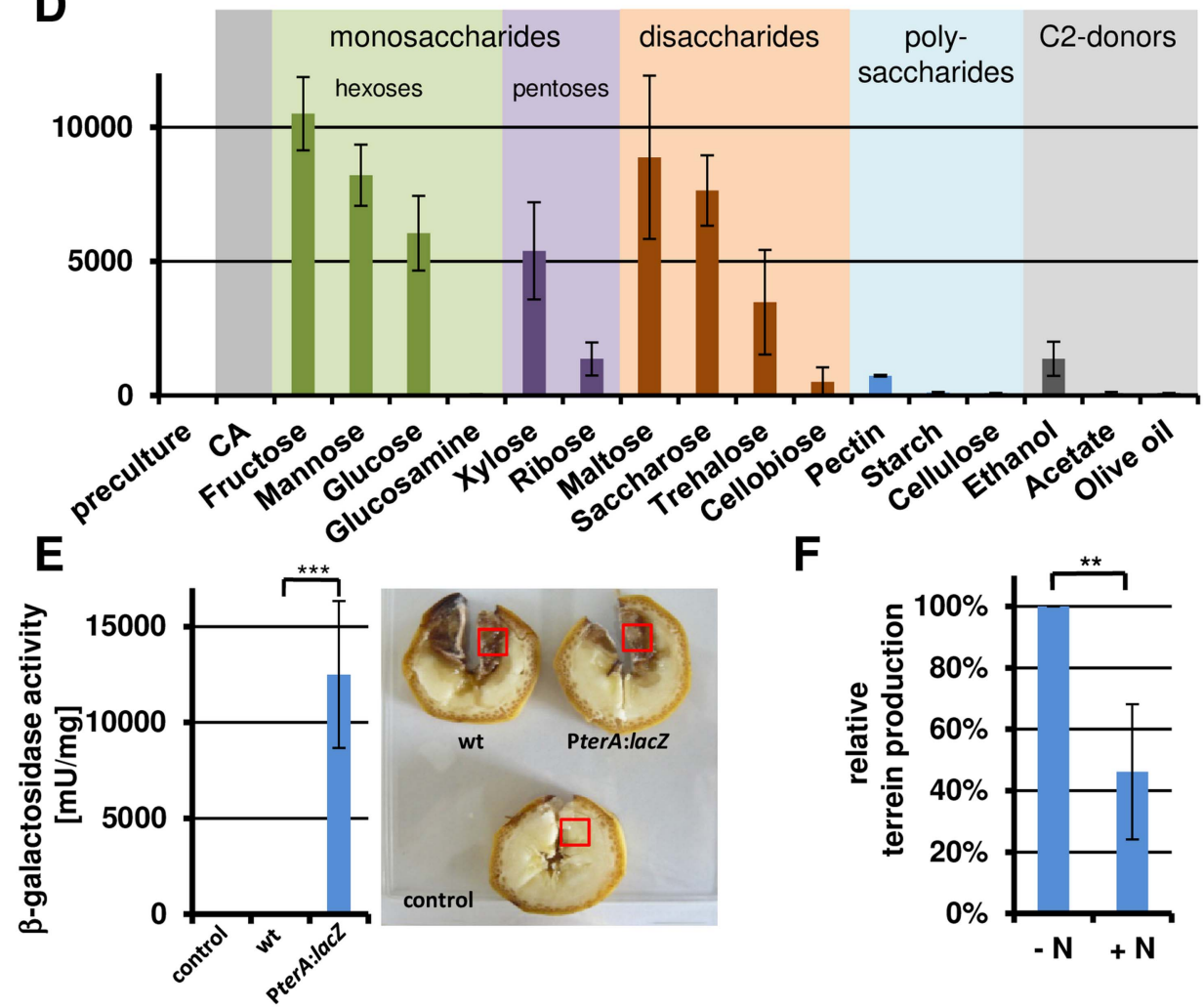

$\mathbf{F}$

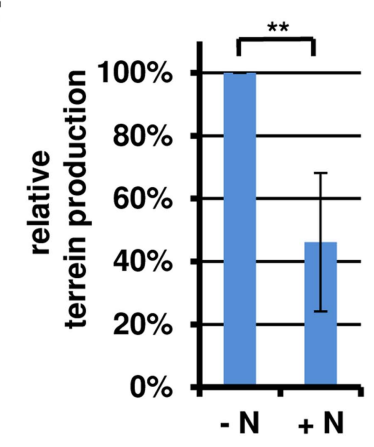

Figure 3. Terrein biosynthesis gene cluster activation under nitrogen starvation. (A) $\beta$-Galactosidase activity of SBUG844_PterA:lacZ was cultivated for 24, 36, 48, 72, and $96 \mathrm{hr}$ in glucose minimal medium supplemented with different concentrations of various nitrogen sources: $70 \mathrm{mM}$ and $10 \mathrm{mM} \mathrm{NaNO}$ or $\mathrm{NH}_{4} \mathrm{Cl}, 1 \%$ or $0.1 \%$ of casamino acids (CA), and 50 or $10 \mathrm{mM}$ aspartate (Asp), asparagine (Asn), glutamate (Glu), or glutamine (Gln). (B) Correlation of Figure 3. continued on next page 
Figure 3. Continued

nitrogen consumption and terA promoter activity determined by $\beta$-galactosidase activity and ammonia consumption of SBUG844_PterA:lacZ in glucose minimal medium with $10 \mathrm{mM} \mathrm{NH}{ }_{4} \mathrm{Cl}$. (C) $\beta$-Galactosidase activity of

SBUG844_PterA:lacZ in nitrogen shift experiments. Cultures grown for $48 \mathrm{hr}$ in glucose minimal medium with $70 \mathrm{mM}$ $\mathrm{NH}_{4} \mathrm{Cl}(\mathrm{PC})$ were washed and transferred to medium with $(+\mathrm{N})$ or without $70 \mathrm{mM} \mathrm{NH}_{4} \mathrm{Cl}(-\mathrm{N})$ and promoter activity was determined after $15 \mathrm{hr}$ of cultivation. (D) Carbon source dependent terA promoter activation under nitrogen starved conditions. Strain SBUG844_PterA:lacZ was pre-cultured for $48 \mathrm{hr}$ on casamino acids without sugar supplementation. The mycelium was washed and transferred to nitrogen-free media with different carbon sources. Reporter activity was determined $24 \mathrm{hr}$ after the shift. (E) $\beta$-Galactosidase activity from bananas infected with conidia suspension of SBUG844 wild-type and PterA:lacZ. Sections (red boxes) were cut from bananas, ground to a fine powder and subjected to $\beta$-galactosidase activity determination. Activity was only detected from the reporter strain. (F) Quantification of terrein from wild-type infected bananas with or without ammonium supplementation. All tests were performed in biological triplicates and technical duplicates; $p$ values were calculated by the Student's paired t-test with a two-tailed distribution: ${ }^{\star *} p<0.01$.

DOI: 10.7554/eLife.07861.008

regulator CpcA (ATEG_03131) (Hoffmann et al., 2001; Krappmann et al., 2004), the stress response bZIP transcription factor AtfA (ATEG_04664) (Balazs et al., 2010; Lara-Rojas et al., 2011), and the nitrogen starvation-induced ras-protein RhbA (ATEG_09480) (Panepinto et al., 2002) were selected for gene deletions. All mutants were tested for their growth properties in the presence of proteinogenic amino acids or ornithine, citrulline, urea, and casamino acids, all of which were used as the sole nitrogen source. Additionally, several complex media were analysed (Figure 4-figure supplement 1).

Only minor growth defects were observed with the cpcA and rhbA mutants, with some general growth reduction on selected amino acids (Asp, His, Ser, Thr) or complex media. However, among all the mutants tested, the areA mutant revealed the most severe growth defects. This mutant grew like the wild-type on glutamine and displayed some reduced growth on the nitrogen-rich amino acids asparagine, aspartate, and histidine as well as on urea and ammonium chloride. However, the $\Delta$ are $A$ strain was unable to use any other proteinogenic amino acid. As described for other Aspergillus species (Hunter et al., 2014), these results confirm an essential role of AreA in nitrogen sensing and utilisation in A. terreus. In contrast, the atfA mutant was only impaired in growth at high aspartate concentrations $(50 \mathrm{mM})$. However, conidia of this mutant lacked the typical yellow-brown pigmentation. Additionally, the atfA mutant completely lacked the typical red colouration of the medium in the presence of methionine, which has previously been demonstrated to be associated with terrein production (Zaehle et al., 2014). This implied a regulatory role of atfA on SM production in A. terreus. On the contrary, the atfA mutant did not show increased sensitivity against oxidative or osmotic stress as described for other Aspergillus species (Balazs et al., 2010; Lara-Rojas et al., 2011). Finally, a double deletion of the are $A$ and atfA genes was generated ( $\triangle \operatorname{are} A \Delta$ atf $A$ ) resembling the growth phenotypes of both single mutants, since it was only able to grow on the media that supported growth of the areA mutant where it formed white conidia, as observed for the $\triangle$ atfA strain.

\section{The global transcription factors AreA and AtfA are essential for terrein biosynthesis gene cluster induction during nitrogen starvation}

To test the effect of transcription factor mutations on terrein biosynthesis gene cluster activation, all mutants were pre-grown on non-inducing glucose medium with glutamine and transferred to medium with or without nitrogen. Terrein was quantified after $24 \mathrm{hr}$ (Figure 4A). The wild-type, the $\Delta c p c A$, and the $\triangle r h b A$ mutant revealed low terrein production in the presence of nitrogen, but high terrein titers when nitrogen was omitted. This indicates that cpcA and rhbA are dispensable for terrein production. In contrast, both the $\triangle$ are $A$ and the $\triangle$ atfA mutant only produced marginal amounts of terrein under nitrogen-limited conditions (Figure 4A), and this effect was completely cured in the complemented mutants are $A^{c}$ and atf $A^{c}$. No terrein was detected in a $\Delta$ are $A \Delta$ atf $A$ double mutant. Therefore, both are $A$ and atfA appear essential for terrein production under nitrogen limitation. To confirm this assumption, we expressed the cluster-specific transcription factor gene terR under control of the gpdA promoter in a $\Delta$ are $A \Delta$ atfA mutant background. A constitutive terrein production was observed, indicating that AreA and AtfA regulate terR expression, but are not essential for expression of the structural genes which directly depend on TerR (Figure 4A). Second, we analysed the effect of areA and atfA deletion on gene 


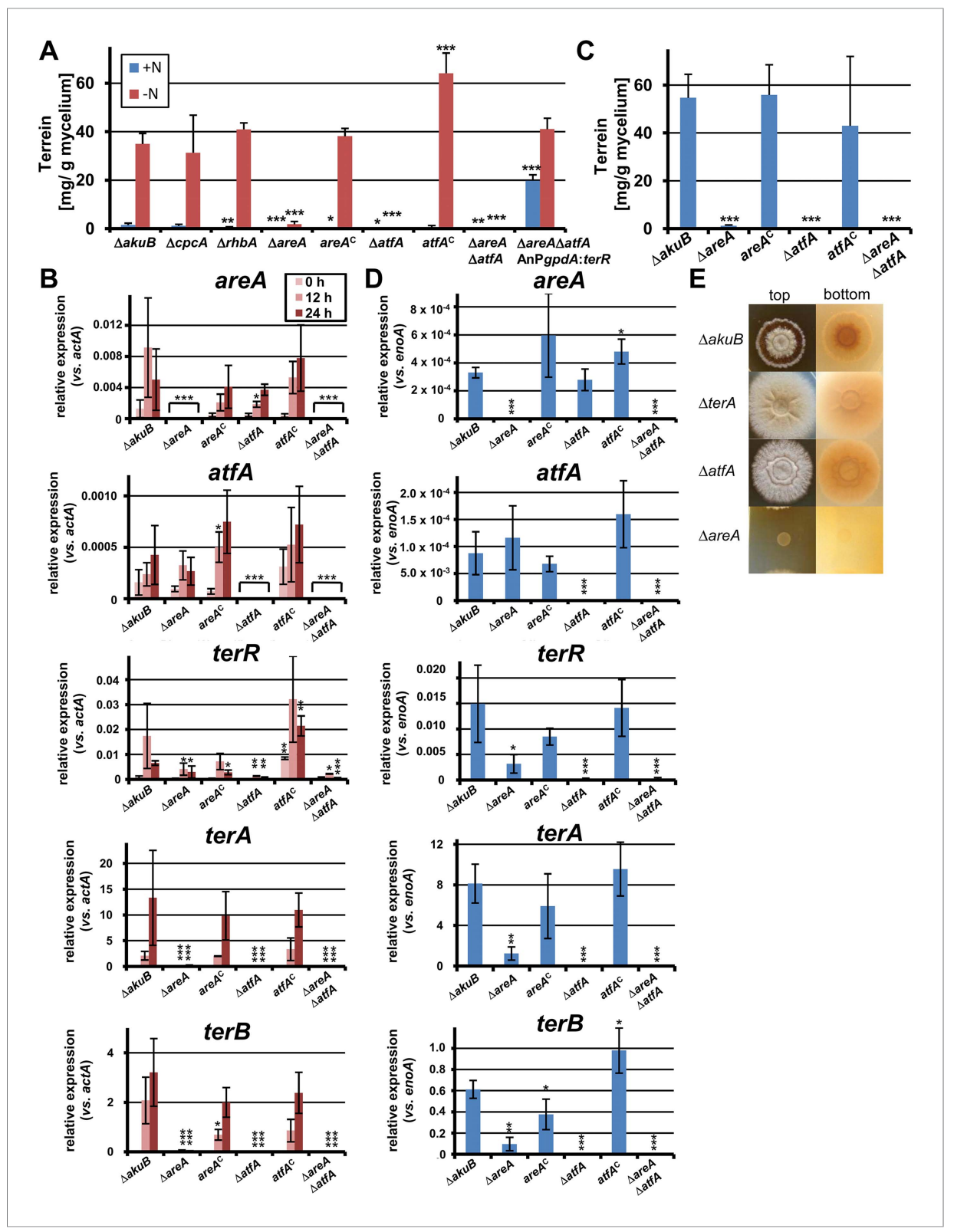

Figure 4. Terrein quantification and expression of terrein cluster genes in nitrogen regulator mutants. (A) Terrein quantification from the parental strains SBUG844 $\Delta a k u B(\Delta a k u B)$, regulator mutants $(\Delta c p c A),(\Delta r h b A),(\Delta a r e A),(\Delta a t f A)$,

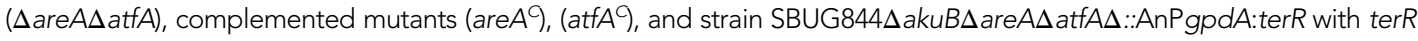
overexpression in the $\Delta$ areA $\Delta$ atfA background. Mycelia were pre-grown in glutamine-supplemented media, washed and transferred to minimal medium with $(+\mathrm{N})$ or without $(-\mathrm{N}) 50 \mathrm{mM}$ glutamine. Terrein was quantified from supernatants $24 \mathrm{hr}$ after the shift. (B) qRT-PCR of strains $\Delta a k u B, \Delta$ areA, are $A^{C}, \Delta$ atfA, atfA $A^{C}$, and $\Delta$ areA $\Delta$ atfA were pre-cultivated for $40 \mathrm{hr}$ in glutamine-supplemented media and transferred to nitrogen starvation. RNA was isolated at 0, 12, and $24 \mathrm{hr}$ of starvation. Transcript levels were normalised against the actin gene act $A$ by fold expression $=2\left(C_{T}^{\text {target }}-C_{T}^{\text {actA }}\right)$.

(C) Terrein quantification from strains shown in (B) after $72 \mathrm{hr}$ of cultivation in glutamine-containing minimal medium supplemented with $10 \mathrm{mM}$ methionine. (D) qRT-PCR from RNA of strains shown in (C) isolated after $48 \mathrm{hr}$ of cultivation. Transcript levels were normalized against the enolase gene enoA by fold expression $=2\left(C_{T}{ }^{\text {target }}-C_{T}{ }^{\text {enoA }}\right)$. $(E)$ Top and bottom view of colonies of Aspergillus terreus wild-type ( $\Delta$ akuB) and mutants ( $\Delta$ terA, $\Delta$ atfA, $\Delta$ are $A)$ grown for $72 \mathrm{hr}$ on solid minimal media supplemented with $25 \mathrm{mM}$ methionine as sole nitrogen source. The red pigmentation of the wildtype (bottom view) is lost in the $\Delta$ terA and $\Delta$ atfA mutants that show some enhanced growth while unable to produce terrein. The $\Delta$ areA strain is unable to grow. In all experiments biological triplicates with technical duplicates were analysed. Statistical significances in comparison to the parental $\Delta a k u B$ strain were calculated by the Student's paired t-test with a two-tailed distribution: ${ }^{\star} \mathrm{p}<0.05,{ }^{*} \mathrm{p}<0.01 ;{ }^{* \star} \mathrm{p}<0.001$

DOI: 10.7554/eLife.07861.009

Figure 4. continued on next page 
Figure 4. Continued

The following source data and figure supplements are available for figure 4:

Source data 1. Genotypes of strains used in the study.

DOI: 10.7554/eLife.07861.010

Source data 2. List of oligo nucleotides used in the study. DOI: 10.7554/eLife.07861.011

Figure supplement 1. Analysis of colony formation and growth phenotypes of nitrogen regulator mutants in the presence of different nitrogen sources DOI: 10.7554/eLife.07861.012

Figure supplement 2. Biochemical characterisation of the recombinant AreA DNA-binding domain and in vitro binding to HGATAR motifs identified in the terR promoter of the Aspergillus terreus terrein biosynthesis gene cluster.

DOI: 10.7554/eLife.07861.013

Figure supplement 3. High performance liquid chromatography analyses from culture filtrates of SBUG844 wildtype and two independent atfA overexpression mutants (AnPgpdA:atfA; OE 1 and 2).

DOI: 10.7554/eLife.07861.014

Figure supplement 4. Southern blot analyses for Aspergillus terreus strains generated in this study. DOI: 10.7554/eLife.07861.015

Figure supplement $\mathbf{5}$. Terrein production and susceptibility to osmotic stress in a sakA mutant. DOI: 10.7554/eLife.07861.016

expression by qRT-PCR from strains shifted for $0 \mathrm{hr}, 12 \mathrm{hr}$, and $24 \mathrm{hr}$ to nitrogen starvation. qRT-PCR was performed on the cluster genes ter $A$, ter $B$, the specific activator ter $R$, and the global transcription factors are $A$ and atfA (Figure 4B).

In the wild-type the regulators are $A$ and atfA showed a time-dependent increase in gene expression. While the regulator terR was most strongly upregulated after $12 \mathrm{hr}$, expression of the TerR-controlled terrein biosynthesis genes terA and terB continued to increase after $12 \mathrm{hr}$ and reached 13.3 and 3.2 times the expression level of the actin control gene at $24 \mathrm{hr}$ (Figure 4B). On the contrary, while complemented mutants behaved like the wild-type, the are $A$ and atfA deletions strongly reduced activation of terR and, in turn, the expression of ter $A$ and ter $B$. Results from QRT-PCR perfectly coincided with the substantially reduced terrein production rates under nitrogen limitation in these mutants (Figure 4A). AreA recognises the DNA-binding motif HGATAR, and two adjacent binding sites are generally required for transcriptional activation due to dimer formation of AreA monomers (Ravagnani et al., 1997). In this respect, the terR promoter contains two putative AreA binding sites that match the HGATAR consensus (BS1and BS2; positions -59 and -72 relative to the translational start point). Surface plasmon resonance (SPR) analyses with the Aspergillus nidulans AreA DNA-binding domain, which is $91 \%$ identical to the respective $A$. terreus AreA domain, showed that BS1 and BS2 are recognised with high affinity (Figure 4-figure supplement 2). This strengthens the model of a direct involvement of AreA in the activation of terR expression. However, the reduction of terR expression was less pronounced in the $\triangle a r e A$ than in the $\triangle$ atfA background (Figure $4 B$ ), which indicates that AreA is not the only activator acting on the terR promoter. Therefore, we additionally searched for putative palindromic AtfA/Sko1 binding sites (5'-TKACGTMA-3') in the promoter regions of the cluster (Proft et al., 2005). Only one hit (TGACGTCA) was identified in the promoter of the structural gene terC. However, if one mismatch is allowed, there is a putative binding site at position -731 relative to the ATG start codon of terR ( $5^{\prime}-$ TGGCGTCA-3'), but it remains speculative whether this binding site is recognised by $A$. terreus AtfA. Nevertheless, it should be mentioned that even a single half site of the suggested motif could promote transcription factor binding and promoter induction (Proft et al., 2005). We therefore conclude that, although direct evidence for AtfA binding at the terR promoter is lacking, both transcription factors seem to regulate terR expression. In agreement, ter $R$, ter $A$, and ter $B$ expression and terrein production showed the strongest decrease in the $\triangle a r e A \Delta a t f A$ double knock-out mutant (Figure 4A). In addition, a constitutive expression of atfA by the gpdA promoter led to increased terrein production (Figure 4-figure supplement 3). This supports our hypothesis of direct involvement of AtfA in terrein cluster regulation. 


\section{AreA and AtfA mediate terrein biosynthesis gene cluster induction in the presence of methionine}

Due to the requirement for atfA and are $A$ in terrein biosynthesis gene cluster induction under nitrogen starvation, we also tested their contribution to methionine-dependent induction in nitrogensupplemented medium (Figure 4C). While the wild-type strain and complemented mutants showed high terrein production rates of up to $55 \mathrm{mg} / \mathrm{g}$ mycelium, the terrein levels in the $\Delta$ are $A, \Delta$ atf $A$, or $\Delta$ are $A \Delta$ atfA double mutant remained near the detection limit and qRT-PCR was performed to confirm this result on the transcriptional level.

Although areA and atfA were expressed in the wild-type only at low levels on glutamine/ methionine medium (Figure 4D), deletion of areA or atfA reduced terR and, consequentially, ter $A$ and ter $B$ expression. In the $\triangle$ atfA mutant terR transcription was completely abolished and, in agreement, transcription of ter $A$ and ter $B$ was no longer detected. An are $A$ mutant is unable to use methionine as a nitrogen source (Figure 4E), and its uptake may be limited leading to the loss of transcriptional activation. In contrast, the atfA mutant still uses methionine as a nitrogen source, but neither produces terrein nor pigmented conidia nor the red colouration of the medium which is associated with terrein production in the presence of methionine (Figure 4E). This implies that atfA may be induced by a methionine-dependent signaling cascade that subsequently leads to terR expression.

\section{Iron limitation acts as a third independent signal for terrein cluster induction}

While nitrogen starvation stimulated terrein production, starvation of other macroelements such as carbon, sulphur, or phosphate did not result in terrein production (data not shown). However, this did not exclude limitation of trace elements as inducing signals. Therefore, the $\beta$-galactosidase activity from the PterA:lac $Z$ reporter strain was determined from cultures with reduced amounts of trace elements. Indeed, a decrease in trace elements was accompanied by increased terA promoter activity (Figure 5A). To attribute this activation to a specific trace element, media with limited amounts of trace elements were supplemented with each of the single trace elements $\mathrm{FeSO}_{4}, \mathrm{ZnSO}_{4}, \mathrm{CuSO}_{4}, \mathrm{MnCl}_{2}, \mathrm{Na}_{2} \mathrm{MoO}_{4}, \mathrm{CoCl}_{2}$, and $\mathrm{H}_{3} \mathrm{BO}_{3}$. Cluster induction was observed in all cultures except for that supplemented with $\mathrm{FeSO}_{4}$ (Figure 5B), indicating cluster activation from iron limitation. Subsequently, a minimal medium was prepared that contained all trace elements but iron which led to strong cluster induction, and the addition of $40 \mu \mathrm{M} \mathrm{FeCl}_{3}$ completely repressed the induction (Figure 5C). Thus, besides methionine and the limitation of nitrogen, a lack of iron-but no other trace element-induces the terrein biosynthesis gene cluster.

\section{Iron starvation induces iron assimilation pathways}

Fungal iron acquisition has been well investigated in A. nidulans (Eisendle et al., 2003) and Aspergillus fumigatus (Haas, 2014), but only limited information was available on the iron acquisition systems from $A$. terreus. A BLASTp search for orthologous genes in the genome of $A$. terreus $\mathrm{NIH} 2624$ (Table 1) revealed that all genes required for a reductive iron assimilation (RIA) system (fetC, fre $B, f \operatorname{tr} A$ ), siderophore biosynthesis (sid genes, except sidG), siderophore transport (mirA- $D, \operatorname{sit} A$ ), and regulators of iron homeostasis ( $\operatorname{srb} A$, sre $A$, and hapX) are well conserved in the $A$. terreus genome. When tested under iron limitation, qRT-PCR analyses confirmed a strong induction of genes from siderophore biosynthesis, siderophore transport, and the RIA pathway (Figure 5-figure supplement 1). To confirm siderophore production by $A$. terreus, we cultivated the wild-type strain under iron-rich $(200 \mu \mathrm{M} \mathrm{FeCl}$ ) and iron-limited conditions (no or $20 \mu \mathrm{M} \mathrm{FeCl}$ ) and identified ferrichrysin (2) and coprogen (3) only under iron limitation (Figure 5D and Figure 5-source data 1, 2) (Zähner et al., 1963; Bertrand et alı, 2010). Coprogen revealed a higher stability than ferrichrysin. Subsequent analyses involving siderophores were therefore based only on coprogen. When terrein was compared with purified coprogen for chelating iron, only coprogen showed the expected iron chelating activity in CAS agar plate assays (Figure 5-figure supplement 2). Thus, although terrein is produced under iron limitation, it does not depict a new kind of siderophore.

\section{Terrein reduces ferric to ferrous iron by its oxidation to propylene maleic acid}

Since some antioxidative properties of terrein had previously been described (Trabolsy et al., 2014), the ability of terrein in reducing ferric $\left(\mathrm{Fe}^{3+}\right)$ to ferrous $\left(\mathrm{Fe}^{2+}\right)$ iron was investigated by using the ferrous 


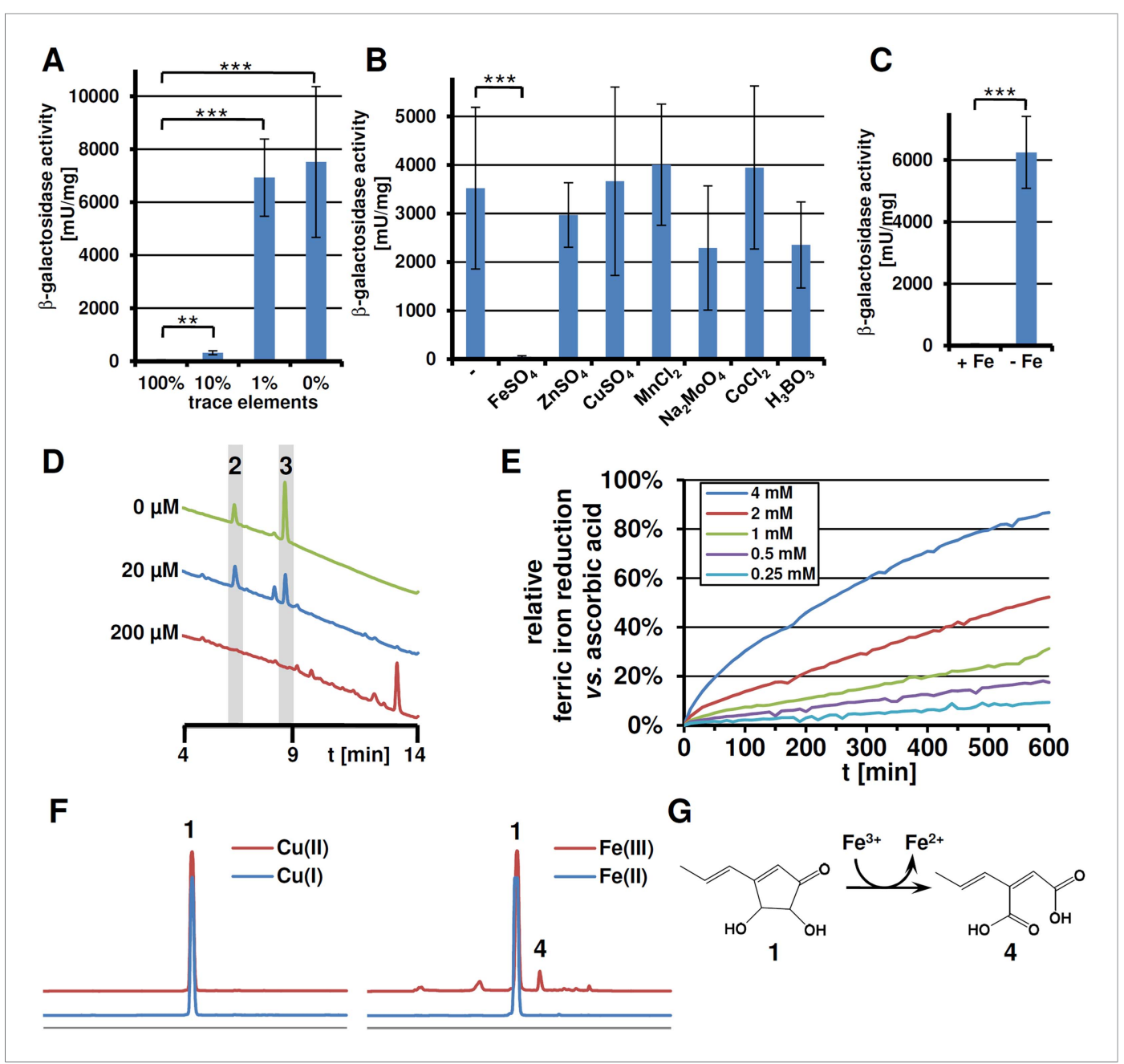

Figure 5. Expression of terA, production of siderophores under iron limited conditions and iron reducing properties of terrein. All analyses were performed in biological triplicates and technical duplicates. Statistical significances were calculated by the Student's paired t-test with a two-tailed distribution. (A) $\beta$-Galactosidase activity from SBUG844_PterA:lacZ in AMM(-N)G100GIn50 medium with limited amounts of trace elements. Asterisks indicate $p$ values vs $100 \%$ trace elements: ${ }^{* *} p<0.01 ;{ }^{* \star *} p<0.001$. (B) $\beta$-Galactosidase activity from SBUG844_PterA:lacZ in AMM(-N)G100G $\ln 50$ medium with $1 \%$ trace elements supplemented with $20 \mu \mathrm{M}$ of specific trace elements: $\mathrm{FeSO}_{4}$, $\mathrm{ZnSO}_{4}, \mathrm{CuSO}_{4}, \mathrm{MnCl}_{2}, \mathrm{Na}_{2} \mathrm{MoO}_{4}, \mathrm{CoCl}_{2}$, or $\mathrm{H}_{3} \mathrm{BO}_{3}$. Asterisks indicate $\mathrm{p}$ values vs activity without supplementation: ${ }^{* * *} \mathrm{p}<0.001$. (C) $\beta$-Galactosidase activity from SBUG844_PterA:IacZ in AMM(-N)G100GIn50 medium with and without $40 \mu \mathrm{M} \mathrm{FeCl}_{3}$. Asterisks indicate $\mathrm{p}$ values vs $40 \mu \mathrm{M} \mathrm{FeCl}_{3}:{ }^{* * *} \mathrm{p}<0.001$. (D) High performance liquid chromatography (HPLC) profiles of lyophilised culture supernatants of $\Delta$ terA after $72 \mathrm{hr}$ of cultivation in AMM(-N)G100G $\ln 50$ supplemented with 0, 20, or $200 \mu \mathrm{M} \mathrm{FeCl}_{3}$. Peaks for the siderophores ferrichrysin (2) and coprogen (3) are indicated. (E) Fe(III) reduction by terrein determined by the TPTZ assay. Ascorbic acid served as control and maximum reduction by ascorbic acid was set as $100 \%$. Analyses were made from duplicates. (F) HPLC profiles of terrein after incubation with different oxidised (upper, red lines) or reduced (lower, blue lines) metal ions. $\mathrm{Left:} \mathrm{Cu}(\mathrm{II}) \mathrm{SO}_{4}$ and $\mathrm{Cu}(\mathrm{I})$ Ac; right: $\mathrm{Fe}(\mathrm{III}) \mathrm{Cl}_{3}$ and $\mathrm{Fe}(\mathrm{II}) \mathrm{SO}_{4}$. $\mathbf{1}$ = terrein; $\mathbf{4}=$ propylene maleic acid. (G) Scheme of terrein oxidation during iron reduction leading to the formation of propylene maleic acid.

DOI: 10.7554/eLife.07861.017

The following source data and figure supplements are available for figure 5:

Source data 1. Analytical data of coprogen.

DOI: 10.7554/eLife.07861.018

Source data 2. Analytical data of ferrichrysin.

DOI: 10.7554/eLife.07861.019

Figure supplement 1. qRT-PCR expression analysis of genes from iron acquisition systems under ironsupplemented and limited conditions.

DOI: 10.7554/eLife.07861.020

Figure 5. continued on next page 
Figure 5. Continued

Figure supplement 2. Chrome azol S (CAS) assay of coprogen and terrein. DOI: 10.7554/eLife.07861.021

Figure supplement 3. $\mathrm{pH}$ - and time-dependent Fe(III) reduction by terrein assayed by TPTZ. DOI: 10.7554/eLife.07861.022

Figure supplement 4. ${ }^{1} \mathrm{H}$ NMR (500 MHz, MeOD; upper panel) and ${ }^{13} \mathrm{C} \mathrm{NMR}$ (150 MHz, MeOD; lower panel) of compound 4, 2-((E)-prop-1-en-1-yl)maleic acid.

DOI: 10.7554/eLife.07861.023

Figure supplement 5. HSOC NMR (600 MHz, MeOD; upper panel) and HMBC NMR (600 MHz, MeOD; lower panel) of compound 4, 2-((E)-prop-1-en-1-yl)maleic acid.

DOI: 10.7554/eLife.07861.024

iron chelator 2,4,6-tripyridyl-S-triazine (TPTZ). The strong antioxidant ascorbic acid (Elmagirbi et al., 2012) served as control (Figure 5E). Although ascorbic acid showed a much higher reducing potential, terrein was also able to convert ferric to ferrous iron in a concentration-dependent (Figure 5E) and $\mathrm{pH}$-dependent (Figure 5-figure supplement 3) manner with a pH optimum of 3-4. In this respect, we noticed a significant fall in the culture $\mathrm{pH}$ under iron limitation from 6.5 to about 3.5, which agrees with the optimum $\mathrm{pH}$ for terrein-mediated iron reduction. Thus, since terrein is produced during growth under iron limitation, the combination with a fall in $\mathrm{pH}$ could indeed increase iron availability.

To elucidate the structure of the terrein oxidation product, the terrein-mediated iron reduction assay was scaled up and extractions were subjected to high performance liquid chromatography (HPLC) analysis. Here, besides a major proportion of terrein that remained in its original structure (Figure 5F), a new peak (4) was observed, which was identified by NMR analyses (Figure 5-figure supplements 4, 5) as 2-((E)-prop-1-en-1-yl) maleic acid (PMA, Figure $5 G)$. The structure of the oxidation product implies that oxidation took place at the two hydroxylated carbon atoms of terrein, leading to a cleavage of the pentenone ring system as proposed by Trabolsy et al. (2014). In contrast, conversion of terrein to PMA was not observed during incubation with $\mathrm{Fe}^{2+}$, confirming that iron reduction was the cause of PMA formation. No conversion of terrein was observed with oxidised copper ions $\left(\mathrm{Cu}^{2+}\right)$, indicating a limited reductive potential which is sufficient for iron but not for copper (Figure 5F).

In conclusion, while terrein cannot chelate iron to support the siderophore-mediated iron uptake, its reductive potential is sufficient to reduce ferric to ferrous iron, which may increase iron solubility and could ease the direct uptake of iron via the ferrous iron transport system.

\section{The iron response regulator HapX regulates siderophore and terrein biosynthesis in A. terreus}

Due to the co-regulation with siderophore biosynthesis, we assumed that transcriptional regulators involved in iron homeostasis could also regulate terrein biosynthesis. In $A$. nidulans and $A$. fumigatus, siderophore biosynthesis is regulated by HapX, a transcriptional inducer under iron limitation, and SreA, a repressor in the presence of iron (Haas, 2012).

To investigate the impact of SreA and HapX on siderophore (coprogen) and terrein synthesis, the sreA gene was completely and the hapX gene partially deleted (the latter due to incomplete sequence information at the hapX locus tag ATEG_08014). Additionally, while the sreA mutant was complemented with the $A$. terreus sreA gene, the hapX mutant was complemented with the hapX gene from $A$. nidulans FGSC A4. All strains were cultivated in iron-supplemented and iron-limited media and terrein and coprogen were quantified from culture supernatants (Figure 6A,B). All complemented mutants behaved similar to the wild-type with production of only marginal amounts of coprogen and terrein in the presence of iron. Under iron starvation the production rates for both metabolites strongly increased.

On the other hand, partial hapX deletion reduced coprogen production under iron limitation by approximately $50 \%$ and terrein concentrations by about $90 \%$ (Figure $6 A, B$ ). This indicates that HapX directly activates both siderophore and terrein biosynthesis in $A$. terreus. In contrast, the $\Delta s r e A$ mutant produced significantly higher amounts of coprogen in the presence of iron whereas terrein production did not significantly increase (Figure $6 A, B$ ). This confirms SreA as a negative feedback regulator in siderophore biosynthesis, whereas it does not control terrein production. In contrast, HapX positively controls both pathways. 
Table 1. BLASTp analysis of homologous genes for iron uptake and regulation of iron homeostasis (adapted by [Haas, 2012])

\begin{tabular}{|c|c|c|c|c|c|}
\hline & $\begin{array}{l}\text { Aspergillus fumigatus } \\
\text { function }\end{array}$ & Gene code $¥$ & Expression & $\begin{array}{l}\text { Aspergillus terreus } \\
\text { gene code }\end{array}$ & Identity/similarity \\
\hline \multicolumn{6}{|c|}{ Reductive iron assimilation (RIA) } \\
\hline FetC & Ferroxidase & AFUA_5G03790 & $-\mathrm{Fe}$ & ATEG_08032 & $79 \% / 89 \%$ \\
\hline FreB & Ferric reductase & AFUA_1G17270 & $-\mathrm{Fe}$ & ATEG_10322 & $53 \% / 64 \%$ \\
\hline FtrA & Iron permease & AFUA_5G03800 & $-\mathrm{Fe}$ & ATEG_08031 & $75 \% / 84 \%$ \\
\hline \multicolumn{6}{|c|}{ Siderophore biosynthesis (SB) } \\
\hline EstA & TAFC esterase & AFUA_3G03660 & $-\mathrm{Fe}$ & ATEG_04072 & $44 \% / 58 \%$ \\
\hline NpgA/PptA & Phosphopantetheinyl transferase & AFUA_2G08590 & - & ATEG_09695 & $56 \% / 65 \%$ \\
\hline SidA & Ornithine monooxygenase & AFUA_2G07680 & $-\mathrm{Fe}$ & ATEG_06879 & $78 \% / 85 \%$ \\
\hline SidC & FC NRPS & AFUA_1G17200 & $-\mathrm{Fe}$ & ATEG_05073 & $60 \% / 76 \%$ \\
\hline SidD & FSC NRPS & AFUA_3G03420 & $-\mathrm{Fe}$ & ATEG_07488 & $43 \% / 59 \%$ \\
\hline SidF & Transacylase & AFUA_3G03400 & $-\mathrm{Fe}$ & ATEG_05075 & $52 \% / 67 \%$ \\
\hline SidG & Transacetylase & AFUA_3G03650 & $-\mathrm{Fe}$ & none & - \\
\hline $\mathrm{SidH}$ & Mevalonyl hydratase & AFUA_3G03410 & $-\mathrm{Fe}$ & ATEG_01509 & $53 \% / 67 \%$ \\
\hline Sidl & Mevalonyl ligase & AFUA_1G17190 & $-\mathrm{Fe}$ & ATEG_05074 & $86 \% / 91 \%$ \\
\hline SidL & Transacetylase & AFUA_1G04450 & - & ATEG_03770 & $64 \% / 76 \%$ \\
\hline \multicolumn{6}{|c|}{ Siderophore transporter (SIT) } \\
\hline MirA & Enterobactin transporter & AN7800; - & $-\mathrm{Fe}$ & ATEG_04071 & $68 \% / 77 \%$ \\
\hline MirB & TAFC transporter & AN8540; -AFUA_3G03640 & $-\mathrm{Fe}$ & ATEG_02711 & $50 \% / 68 \%$ \\
\hline $\mathrm{MirC}^{*}$ & & AN7485; AFUA_2G05730 & $-\mathrm{Fe}$ & ATEG_06762 & $78 \% / 87 \%$ \\
\hline MirD† & Trichotecene efflux pump & AFUA_3G03440 & - & ATEG_07487 & $40 \% / 58 \%$ \\
\hline SitA/SitT* & & AN5378; AFUA_7G06060 & $-\mathrm{Fe}$ & ATEG_06329 & $62 \% / 73 \%$ \\
\hline \multicolumn{6}{|c|}{ Regulatory proteins } \\
\hline HapX & bZip-TF & AFUA_5G03920 & $-\mathrm{Fe}$ & ATEG_08014 & $\begin{array}{l}77 \% / 83 \% \text { (hapX } \\
\text { re-annotated) }\end{array}$ \\
\hline SreA & GATA TF & AFUA_5G11260 & $+\mathrm{Fe}$ & ATEG_07741 & $67 \% / 75 \%$ \\
\hline SrbA† & HLH TF & AFUA_2G01260 & $-\mathrm{Fe}$ & ATEG_08156 & $72 \% / 82 \%$ \\
\hline
\end{tabular}

Genes selected for qPCR analyses are highlighted in bold

*Genes annotated according to (Schrettl et al., 2008)

†Genes annotated according to (Blatzer et al., 2011)

$\neq$ Gene codes ANxxxx refer to the $A$. nidulans genome.

DOI: 10.7554/eLife.07861.025

These results were confirmed on the transcriptional level by qRT-PCR (Figure 6C and Figure 6-figure supplement 1). The inactivation of hapX prohibited terR transcription and resulted in an inability to induce terrein cluster genes ter $A$, ter $B$, and ter $C$. In contrast, deletion of sre $A$ influenced neither terR activation nor expression of other terrein cluster genes in the presence of iron. We therefore conclude that hapX is the major regulator for terrein cluster induction under iron starvation.

To elucidate the complete sequence of the hapX gene we subsequently used degenerate primers to amplify the main proportion of hapX from cDNA. The complete sequence of the $A$. terreus hapX gene is found under accession number KP233834 (Gressler et al., 2015b). The sequence of the fulllength HapX protein matches with that of HapX proteins from other Aspergillus species such as A. nidulans (73\% identity), A. fumigatus (77\%), Aspergillus niger (81\%), and A. oryzae (81\%).

\section{Terrein supports growth in the absence of the siderophore system}

In general, siderophore-based iron acquisition is highly efficient and assumed to be more important than the reductive iron assimilation pathway. In A. fumigatus, growth and virulence defects caused by 


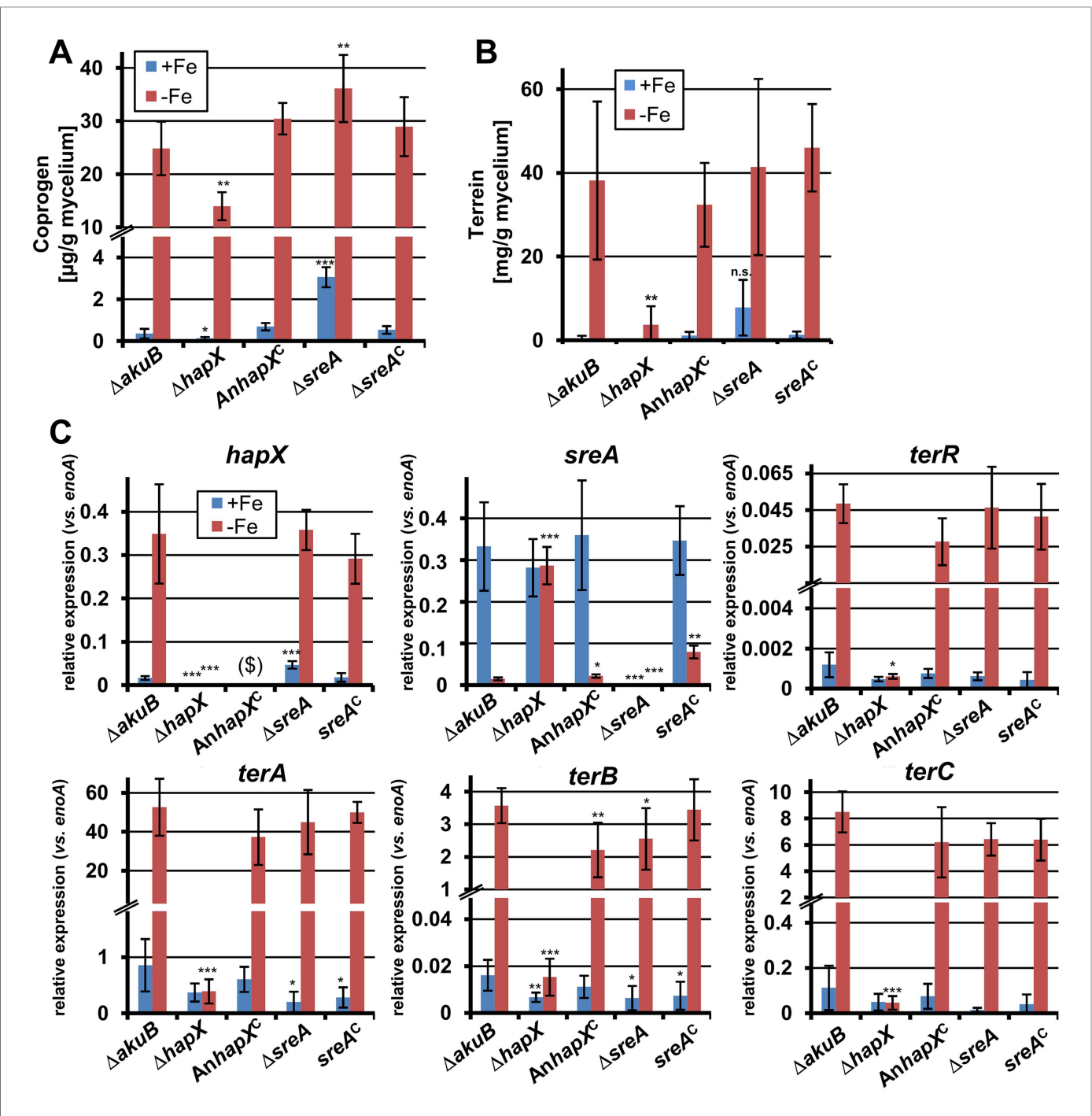

Figure 6. Effect of hapX and sreA deletion on terrein and coprogen biosynthesis. (A) Coprogen or (B) terrein

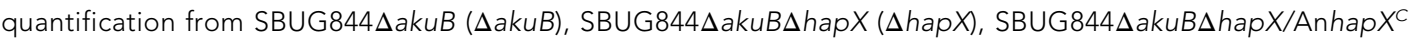

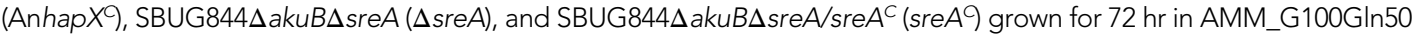
with $(+\mathrm{Fe})$ or without $40 \mu \mathrm{M} \mathrm{FeCl}_{3}(-\mathrm{Fe})$. Coprogen was quantified from lyophilised culture supernatants and terrein from culture extracts. (C) qRT-PCR from strains and media described in (A) and (B). RNA was isolated after $40 \mathrm{hr}$ of cultivation. Transcript levels were normalised against enoA by fold expression $=2\left(C_{T}{ }^{\text {target }}-C_{T}{ }^{\text {enoA }}\right)$. (\$) denotes the lack of hapX transcripts from the complemented $\Delta$ hapX strain, since the Aspergillus nidulans hapX was used for complementation. qRT-PCR on the complemented mutant with oligonucleotides specific for AnhapX are shown in Figure 6-figure supplement 1. All analyses were performed from biological triplicates and technical duplicates. Statistical significances were calculated in comparison to the parental $\Delta a k u B$ strain by the Student's paired t-test with a two-tailed distribution: ${ }^{*} \mathrm{p}<0.05 ;{ }^{* \star} \mathrm{p}<0.01 ;{ }^{* \star *} \mathrm{p}<0.001$.

DOI: 10.7554/eLife.07861.026

The following figure supplement is available for figure 6 :

Figure supplement 1. qRT-PCR analysis of Aspergillus nidulans hapX expression in the Aspergillus terreus wild-type

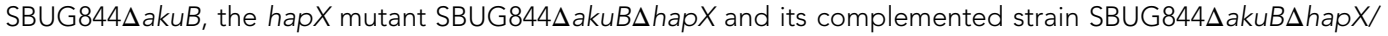
Anhap $X^{C}$.

DOI: 10.7554/eLife.07861.027

the interruption of the reductive iron assimilation pathway are only observed when the siderophore-based system is also inactivated (Blatzer et al., 2011). Therefore, to elucidate a positive effect of terrein on iron acquisition, we deleted the sidA gene in $A$. terreus that encodes the L-ornithine- $N^{5}$-monooxygenase, a key 
enzyme in hydroxamate siderophore biosynthesis. Coprogen production was confirmed in the wild-type and a complemented mutant, but was completely lacking from the $\Delta$ sidA mutant (Figure 7-figure supplement 1). When analysed for growth phenotypes on solid media, all complemented mutants and the $\Delta$ terA strain behaved like the wild-type (Figure 7A and Figure 7-figure supplement 1A). The hapX

A

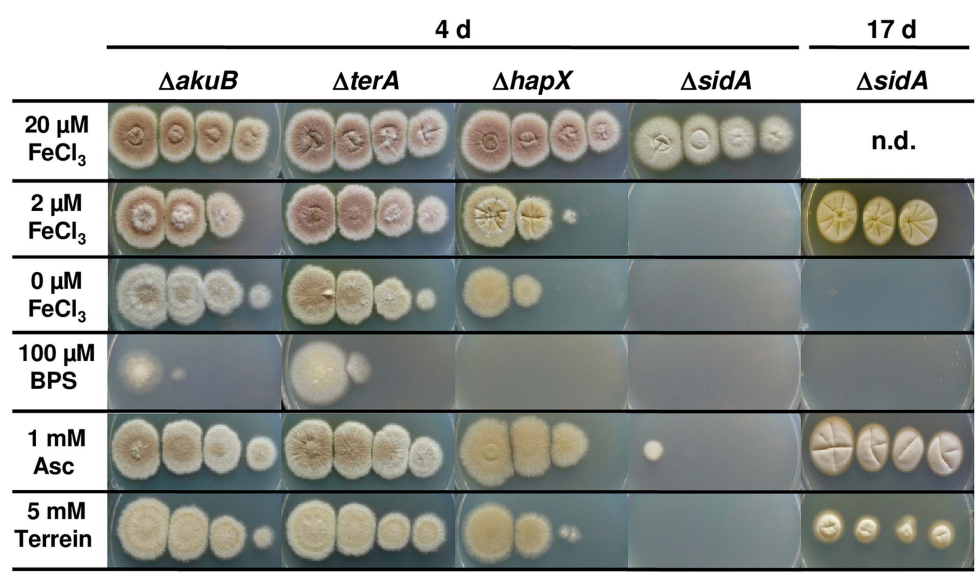

B

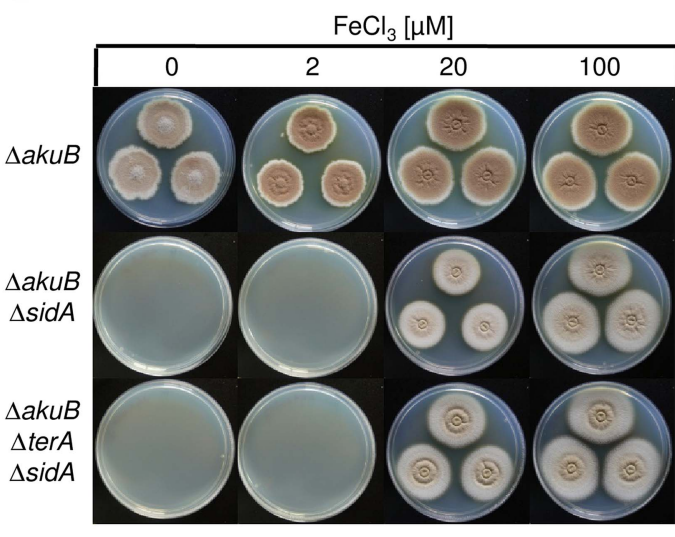

C

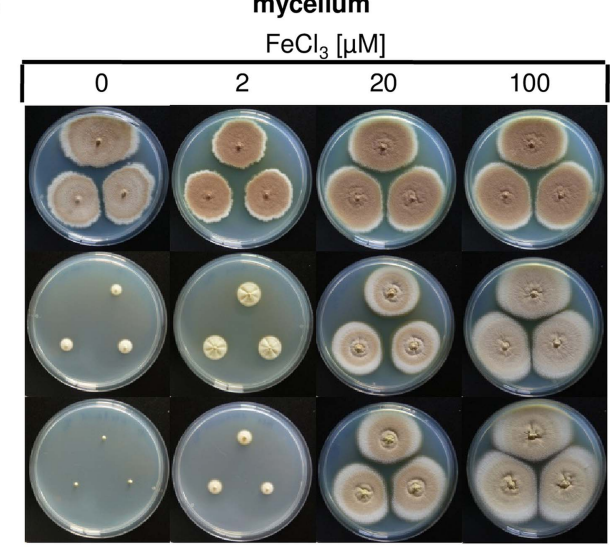

Figure 7. Siderophore production in sidA mutants and growth-supporting effect of terrein under iron limitation. (A) Growth of wild-type $\Delta a k u B, \Delta$ terA, $\Delta$ hapX, and $\Delta$ sidA on AMM(-N)G100G $\ln 10$ plates containing different iron concentrations. Photographs were taken after $4 \mathrm{~d}$ and, as indicated, after $17 \mathrm{~d}$ of incubation at $37^{\circ} \mathrm{C}$. Iron-free medium was supplemented with $100,20,2$, or $0 \mu \mathrm{M} \mathrm{FeCl}$. Additionally, plates without iron were supplemented with bathophenanthroline disulfonate (BPS; $100 \mu \mathrm{M}$ ) to simulate harsh starvation conditions or with either ascorbic acid (1 $\mathrm{mM}$ ) or terrein (5 or $10 \mathrm{mM}$ ). (B, C) Impact of terrein production on growth of $\Delta$ sidA mutants. AMM-G100G $\ln 50$ plates were supplemented with $0,2,20$, or $100 \mu \mathrm{M} \mathrm{FeCl}$ and either inoculated with (B) conidia or (C) mycelial pellets from cultures pre-grown for $40 \mathrm{hr}$ in AMM-G100Gln50 with $200 \mu \mathrm{M} \mathrm{FeCl}_{3}$. The parental Aspergillus terreus wild-type $\Delta a k u B$, the $\Delta a k u B \Delta$ sidA strain, and the $\Delta a k u B \Delta t e r A \Delta$ sidA are shown. Mycelia from a $40 \mathrm{hr}$ culture in AMMG100G In50 with $200 \mu \mathrm{M} \mathrm{FeCl}_{3}$ was washed with iron-free medium and three pellets were applied to the plates. Plates were incubated at $37^{\circ} \mathrm{C}$ for $5 \mathrm{~d}$.

DOI: 10.7554/eLife.07861.028

The following figure supplements are available for figure 7 :

Figure supplement 1. Dependence of colony formation of Aspergillus terreus mutants on iron availabilty, ascorbic acid, terrein, and coprogen.

DOI: 10.7554/eLife.07861.029

Figure supplement 2. Antifungal activity of terrein and potato dextrose broth (PDB) medium from Aspergillus terreus wild-type cultivations.

DOI: 10.7554/eLife.07861.030

Figure supplement 3. Terrein determination in siderophor deletion mutants.

DOI: 10.7554/eLife.07861.031 
mutant showed a reduced growth rate without iron supplementation (Figure 7A), which is in agreement with reduced coprogen production as shown above (Figure 6A). However, severe iron limitation from the addition of the iron chelator bathophenanthroline sulfonate (BPS) completely repressed growth of the hapX mutant (Figure 7A). The $\Delta$ sidA mutant displayed the most severe phenotype: while $\Delta$ sidA showed normal growth with slightly reduced conidiation in the presence of high to moderate iron concentrations (100 or $20 \mu \mathrm{M} \mathrm{FeCl}_{3}$ ) (Figure 7A), growth was strongly retarded when the iron concentration was reduced to $2 \mu \mathrm{M} \mathrm{FeCl}$. No growth was observed even after prolonged incubation when iron was omitted (Figure 7A). These phenotypes were cured when purified coprogen was externally added (Figure 7-figure supplement 1B). Interestingly, growth of the $\Delta$ sidA strain was also partially restored in the presence of ascorbic acid and, although to a lesser extent, by the addition of terrein (Figure 7A). This result is supported by previous studies on an A. nidulans $\Delta$ sidA mutant which was able to grow under iron limitation in the presence of ascorbic acid (Eisendle et alo, 2003). Unexpectedly, terrein supplementation inhibited growth of an A. fumigatus sidA mutant, which was also true for an A. fumigatus wild-type strain (Figure 7-figure supplement 2). Subsequent analyses showed that growth of the phytopathogen Fusarium graminearum was also inhibited by terrein-containing culture extracts from A. terreus (Figure 7-figure supplement 2), indicating some antifungal properties of terrein against environmental competitors.

Due to the beneficial effect of terrein on the $A$. terreus $\Delta$ sidA mutant, we investigated the impact of intrinsic terrein production on growth under iron limitation. For this, we deleted the sidA gene in the $\Delta$ terA background and compared growth of wild-type, $\Delta$ sidA, and $\Delta$ terA $\Delta$ sidA under ironsupplemented ( 20 and $200 \mu \mathrm{M} \mathrm{FeCl}_{3}$ ) and iron-limited conditions ( 0 and $2 \mu \mathrm{M} \mathrm{FeCl}_{3}$ ) (Figure 7B). Both mutants ( $\Delta$ sidA and $\Delta$ terA $\Delta$ sidA) were unable to grow on media containing $2 \mu \mathrm{M}$ or less $\mathrm{FeCl}_{3}$. However, since terrein is only produced by vegetative mycelium, strains were pre-grown in the presence of $200 \mu \mathrm{M} \mathrm{FeCl}$, washed, and transferred to the plates with different iron contents (Figure 7C). While growth of mycelium from the $\triangle a k u B$ strain looked identical to that from conidia, the $\Delta$ sidA mutant started to form small colonies even in the absence of iron, whereas the $\Delta$ terA $\Delta$ sidA mutant showed some weak growth only at $2 \mu \mathrm{M} \mathrm{FeCl}_{3}$ but not in the absence of external iron supplementation. Terrein production was analysed from extracted agar plugs, which confirmed that the wild-type and $\Delta$ sidA mutant produced substantial amounts of terrein under iron-limited conditions whereas terrein production was fully abrogated in the $\Delta$ terA $\Delta$ sidA mutant (Figure 7-figure supplement 3 ). These results indicate that terrein production supports iron acquisition, although the siderophore system is the dominant iron acquisition system.

\section{Discussion}

A. terreus is known as a human pathogen that causes severe invasive bronchopulmonary and disseminated aspergillosis (Lass-Flörl et al., 2005; Slesiona et al., 2012a, 2012b). In addition, A. terreus has been described as a causative agent of foliar blight of potato leaves (Louis et al., 2013, 2014a, 2014b). Besides its pathogenic potential, A. terreus can inhibit proliferation of other plant pathogens such as Fusarium udum (Upadhyay and Rai, 1987) and acts as a mycoparasite on sclerotia of the plant pathogenic fungus Sclerotinia sclerotiorum (Melo et al., 2006).

SMs are assumed to support competition in the plant and soil environment. The natural biological activities of terrein include the inhibition of plant seed germination (Kamata et al., 1983), the induction of fruit surface lesions (Zaehle et al., 2014), and the newly discovered reduction of ferric to ferrous iron accompanied by some antifungal activity. These activities are all appropriate to supporting the competitiveness of $A$. terreus in the environment. However, the production of terrein at the correct timing requires coordinated sensing and transduction of environmental signals.

The terrein biosynthesis gene cluster is directly controlled by the $\mathrm{Zn}_{2} \mathrm{Cys}_{6}$ transcription factor TerR (Gressler et al., 2015a). Therefore, terrein production requires transcriptional activation of terR. While sugars are indispensable for high terrein production rates (Zaehle et al., 2014), three major signals were identified that resulted in terR transcription: methionine-dependent induction, nitrogen limitation, and iron starvation. These signals resemble the plant and rhizosphere environment.

Plant roots are leaky for carbon compounds that derive from C-fixation during photosynthesis. Up to one third of plant-assimilated carbon may end up in the rhizosphere, and especially in the extramatrical mycelium of ectomycorrhiza (Churchland and Grayston, 2014). Here, at least a fraction of carbohydrates is subsequently exuded by ectomycorrhiza, making it available to other soil microorganisms (Sun et alo, 1999). Mycorrhizas mobilize nitrogen to feed their symbiotic plant partner, resulting in 
nitrogen limitation for surrounding microorganisms. These conditions stimulate terrein production in A. terreus, which is typically isolated from the rhizosphere (Gao et al., 2013; Rajalakshmi and Mahesh, 2014) and might affect existing plant-microbe interactions by its phytotoxic and antifungal activity (Figure 8).

Another inducing signal derives from methionine, which may be of particular importance when A. terreus acts as a potato plant pathogen (Louis et al., 2013). Notably, PDB induces high terrein production rates (Zaehle et al., 2014), which we assume to be methionine-dependent. In plants, methionine is required for ethylene production in environmental stress response (Wang et al., 2002), fruit ripening (Yang and Hoffman, 1984), and polyamine production in plant defence reactions. Furthermore, methionine is a precursor for iron chelating siderophores such as nicotianamine and mugineic acid (Roje, 2006) and has been identified in plant root exudates (Dakora and Phillips, 2002). Therefore, elevated levels of methionine depict a signal that indicates the presence of a plant environment and stimulates terrein production. However, although further studies are required to elucidate the specific contribution of terrein to plant infection, its ability to induce lesion formation on several fruit surfaces indicates that terrein interacts with plant defence mechanisms (Zaehle et al., 2014).

The third-at first sight unrelated-inducing factor is iron starvation. Although terrein can reduce ferric to ferrous iron, a direct positive growth-promoting effect of terrein during iron limitation in

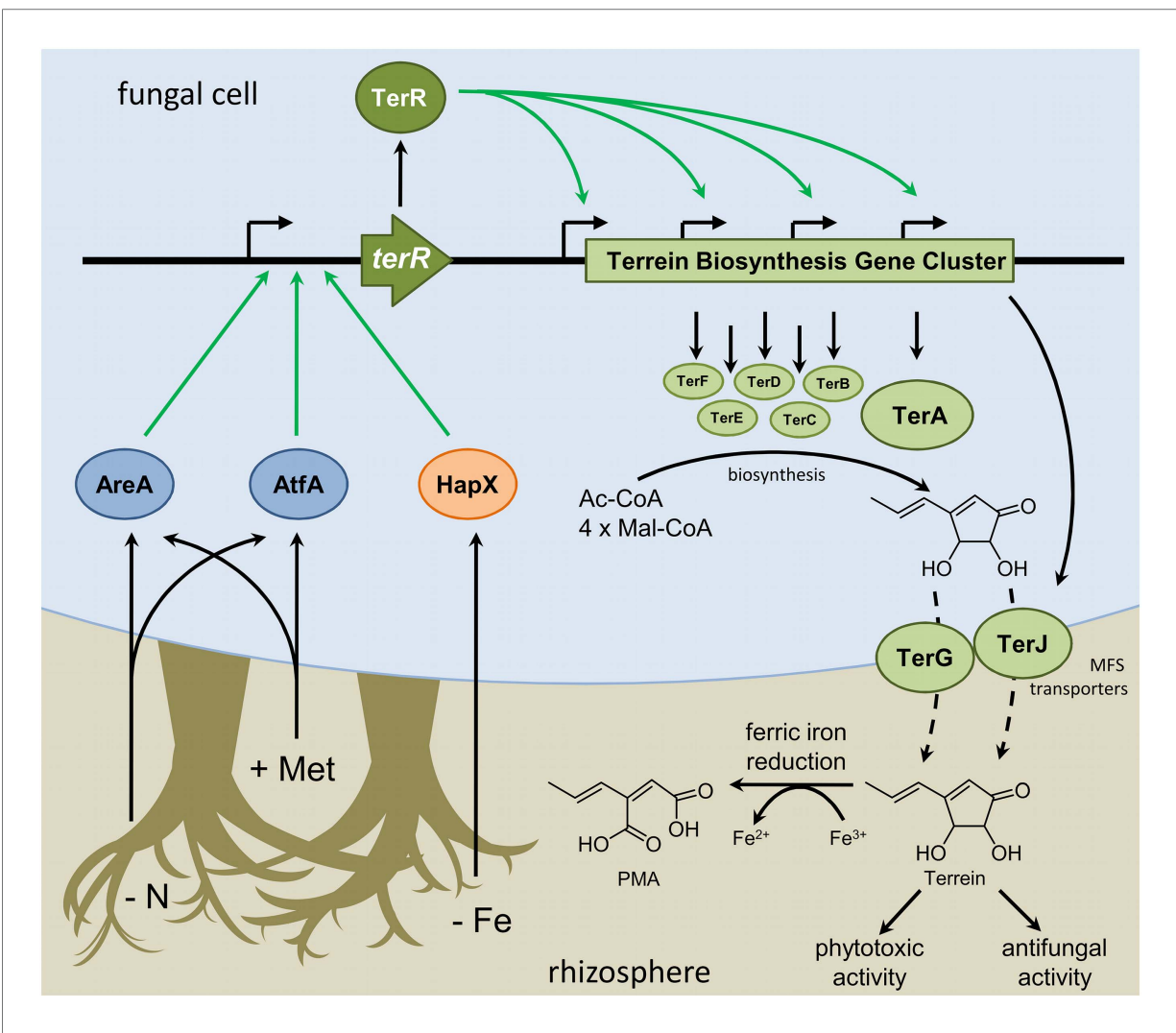

Figure 8. Scheme of the regulation of terrein biosynthesis gene cluster expression during interactions in the rhizosphere. Plants secrete methionine (+Met) with root exudates into the soil. Additionally, competing microorganisms reduce the available pool of nitrogen sources $(-N)$ and iron $(-F e)$. Signals from nitrogen limitation and methionine are sensed via AreA and AtfA, whereas iron limitation is sensed via HapX. All three transcription factors activate the promoter of the terrein biosynthesis gene cluster-specific transcription factor. TerR leads to transcription of the structural genes required for terrein biosynthesis and terrein is produced from acetyl- and malonyl-CoA units. The two-cluster specific major facilitator superfamily (MFS) transporters export terrein into the rhizosphere. Here terrein can counteract iron limitation by its ferric iron reducing activity, supports degradation of organic matter by its phytotoxic activities, and reduces growth of competitors by its antifungal activity.

DOI: 10.7554/eLife.07861.032 
A. terreus monocultures was only observed in the absence of a functional siderophore system. However, extracellular siderophores show a high affinity for ferric iron but not for ferrous iron (Hider and Kong, 2010). Thus, the reduction of ferric iron could reduce the efficiency of iron chelation by xenosiderophores secreted from competing microorganisms. A similar strategy for iron acquisition has previously been described for the human pathogenic fungus Histoplasma capsulatum (Timmerman and Woods, 1999). H. capsulatum secretes glutathione accompanied by the $\gamma$-glutamyltransferase Ggt1. This enzyme releases the dipeptide cysteinylglycine from gluthathione, which in turn reduces extracellular ferric iron (Zarnowski et alo, 2008). While H. capsulatum also secretes hydroxamate siderophores (Howard et al., 2000), silencing of ggt1 mRNA reduced virulence in macrophages even in the presence of the siderophore system (Zarnowski et al., 2008). Thus, terrein may interfere with competing siderophore systems to increase the competitiveness of $A$. terreus in the environment.

In this study we showed that transduction of environmental signals to the promoter of the transcriptional activator TerR requires activation by the global transcription factors AreA, AtfA, and HapX. While AreA has previously been described to regulate several SM gene clusters in fungi (Tudzynski et al., 1999), HapX activates iron acquisition systems (Schrettl et alo, 2010) and AtfA plays a specific role in osmotic and oxidative stress responses (Lara-Rojas et al., 2011).

In Aspergillus species and maybe all saprophytic ascomycetes, the GATA transcription factor AreA plays a substantial role in regulation of nitrogen. Under nitrogen starvation, AreA is transported into the nucleus (Todd et alo, 2005) where it binds a 5'-HGATAR-3' sequence in its target promoters (Ravagnani et al., 1997), as shown for the genes encoding nitrate reductase (NiaD) and nitrite reductase (NiiD) (Chang et alo, 1996) that are upregulated in the absence of preferred nitrogen sources (Johnstone et al., 1990; Punt et al., 1995). In Aspergillus parasiticus AreA also binds to the promoter regions of the transcriptional regulator genes aflR and aflJ from the aflatoxin biosynthesis cluster (Chang et al., 2000). In contrast to nitrate utilisation, this binding impedes aflatoxin production probably due to inhibition of transcriptional activation (Yu et al., 2004). We have shown that terrein biosynthesis in $A$. terreus during nitrogen starvation is AreA-dependent, and at least two are $A$ binding sites in the ter $R$ promoter were recognised by the AreA DNA-binding domain. Therefore, AreA directly binds to the terR promoter and supports the expression of the terrein biosynthesis genes. Similarly, biosynthesis of various mycotoxins and gibberellins is activated by AreA in phytopathogenic Fusarium species (Mihlan et al., 2003; Kim and Woloshuk, 2008; Giese et al., 2013). This implies that AreA plays a general role in regulation of SM production of plant pathogens, and the plant-derived environment may be characterised as a habitat with a high C: $\mathrm{N}$ ratio.

In several ascomycetes the nitrogen starvation response is also mediated by the basic leucine zipper transcription factor AtfA (Hong et al., 2013). In A. nidulans, nitrogen starvation, oxidative or osmotic stresses induce phosphorylation of the MAP kinase SakA that is subsequently transported into the nucleus to activate transcription of target genes by interaction with the constitutively expressed transcription factor AtfA (Lara-Rojas et alo, 2011). Interestingly, in A. terreus the interaction between AtfA and SakA has not been solved in detail. While a sakA deletion does not affect terrein production levels under nitrogen limitation, it allows terrein production in the presence of some nitrogen sources, indicating that it may possess a repressor function under some conditions. In addition, a $\Delta s a k A$ strain still produces pigmented conidia (Figure 4-figure supplement 5) and the sakA mutant-but not the $\triangle$ atfA strain-showed some increased osmosensitivity (Figure 4-figure supplement 5), which agrees with recent data on $\triangle a t f A$ and $\Delta$ sakA mutants of Penicillium marneffei (Nimmanee et al., 2014). Therefore, it is conceivable that terrein biosynthesis gene cluster activation via AtfA does not require SakA.

In A. terreus AtfA seems to act as a general inducer of SM production since its deletion resulted in non-pigmented conidia and in the loss of terrein production under nitrogen starvation or methionine supplementation. In contrast, deletion of the atf1 gene in Botrytis cinerea resulted in accumulation rather than depletion of the polyketide botcinin $A$ and the sesquiterpene botrydial (Temme et al., 2012). This points to AtfA as a transcriptional inhibitor in $B$. cinerea whereas AtfA acts as an inducer in $A$. terreus. Interestingly, AreA and AtfA sense the same environmental signals to induce the terrein biosynthesis gene cluster, although their global role in cellular physiology differs significantly. This adaptation of different transcription factors towards terrein production underlines a special importance of terrein for $A$. terreus during environmental competition.

Independent from AreA and AtfA, activation of the terrein biosynthesis gene cluster was also triggered by the iron response regulator $\mathrm{HapX}$ under iron limitation. To overcome severe iron 
starvation, microorganisms frequently produce siderophores (Pollack and Neilands, 1970; Eisendle et al., 2003; Kreutzer and Nett, 2012) or utilise siderophores from competing species (xenosiderophores) (Boukhalfa and Crumbliss, 2002). In A. fumigatus and A. nidulans siderophore biosynthesis and uptake is regulated by the opposing transcription factors HapX and SreA depending on the presence or absence of iron (Schrettl et al., 2007, 2008, 2010). Similarly, A. terreus produces the HapX and SreA regulated siderophores ferrichrysin and coprogen that allow efficient acquisition of iron. However, in competition with other micoorganisms, terrein-mediated iron reduction could reduce binding of iron to high-affinity siderophores from competitors that cannot be used by $A$. terreus, as described for the cysteinylglycine iron reduction system from $H$. capsulatum.

The control of terrein production by HapX depicts an unprecedented example of the activation of a SM gene cluster outside the siderophore biosynthesis system. Interestingly, terrein production is only under positive HapX control, but not under negative SreA feedback control, which is in agreement with a lack of SreA binding sites in the terR promoter (Schrettl et al., 2008), whereas a CCAAT box required for binding of the CCAAT-binding complex $(\mathrm{CBC})$ and its subsequent $\mathrm{CBC}$ interaction with HapX (Hortschansky et al., 2007; Gsaller et al., 2014) is present. In the HapX target promoter regions of cycA, sreA, acoA, and lys $F$ from Aspergillus species, a bipartite $C B C-H a p X-D N A$ binding motif with consensus $5^{\prime}-\mathrm{C}(\mathrm{C} / \mathrm{A}) \mathrm{AATCAN}{ }_{11-12} \mathrm{GAT}-3^{\prime}$ is present, of which CCAAT is recognised by the CBC complex while the GAT sequence is bound by HapX (Hortschansky et alo, 2015). Interestingly, these are all types of promoters that are repressed under iron limitation. In contrast, the cccA promoter, which encodes for a vacuolar iron transporter, is the only HapX-induced promoter known so far with a confirmed in vitro HapX binding site (Gsaller et al., 2014). HapX binds to the GAT motif of a $5^{\prime}$-CCAATN ${ }_{16}$ GATC-3' sequence, which is also present in the cccA promoter of $A$. terreus and-with one mismatch-in the terR promoter (position -215). However, further studies will be required to confirm this motif as a HapX binding site.

In conclusion, we have shown that the terrein biosynthesis gene cluster is activated by at least three independent environmental signals: methionine, nitrogen limitation, and iron starvation. Additionally, the three global transcription factors AreA, AtfA, and HapX are essential to transfer these signals to the terrein biosynthesis gene cluster. Although one environmental signal is sufficient to induce the gene cluster, a combination of different signals provides an environmental image of higher resolution and allows an adequate adaptation to the ecological niche. The phytotoxic and antifungal potential of terrein combined with its iron reductive properties enables $A$. terreus to acquire nutrients that might otherwise be consumed by competitors. Notably, similar to terrein, the mycotoxin production in Fusarium species is generally associated with sensing of nitrogen availability. Although AreA plays an important regulatory function, involvement of AtfA as an additional regulator and methionine as a second inducer in these species needs to be tested. However, from our results on the terrein biosynthesis gene cluster, we propose that manifold regulation of SM gene clusters and the interplay of different global transcriptional activators might depict a general mechanism to regulate the flexibility of gene cluster activation in fungi.

\section{Materials and methods}

\section{Media and cultivation conditions}

All genotypes of strains used in this study are summarised in (Figure 4-source data 1). A. terreus strain SBUG844 and its derivative SBUG844 $\triangle a k u B$ that show increased rates of homologous integration served as parental strains (Gressler et al., 2011). If not indicated otherwise, all strains were cultivated in Aspergillus minimal medium (AMM; http://www.fgsc.net/methods/anidmed.html) supplemented with different carbon and nitrogen sources: AMM with $100 \mathrm{mM}$ glucose (AMM-G100), with $100 \mathrm{mM}$ glucose and $50 \mathrm{mM}$ glutamine (AMM-G100Gln50), with $100 \mathrm{mM}$ glucose, $50 \mathrm{mM}$ glutamine and $10 \mathrm{mM}$ methionine (AMM-G100G In50Met10), with 1\% casamino acids (AMM-CA1\%). Additionally, the following complex media were used: Sabouraud (Sab, Sigma Aldrich, Taufkirchen, Germany), YPD (10 g/l yeast extract, $20 \mathrm{~g} / \mathrm{l}$ peptone, $20 \mathrm{~g} / \mathrm{l}$ dextrose; PDB, Sigma Aldrich), potato broth (2\% potato extract, Sigma Aldrich), apple juice ( $\mathrm{pH}$ adjusted to 6.0 with $\mathrm{NaOH}$ ), carrot juice with honey (both Wiesgart, ALDI Nord, Jena, Germany), banana juice or peach juice (both FruchtOase, Kiberg, Germany). When required, $140 \mu \mathrm{g} / \mathrm{ml}$ hygromycine B (Carl Roth $\mathrm{GmbH}$, Karlsruhe, Germany), $0.1 \mu \mathrm{g} / \mathrm{ml}$ pyrithiamine or $80 \mu \mathrm{g} / \mathrm{ml}$ phleomycin (both Sigma Aldrich) were added. For preparation of conidia suspensions, all strains were cultivated for $4 \mathrm{~d}$ at $37^{\circ} \mathrm{C}$ on solid $2 \%$ AMM-G50Gln10 agar plates. Conidia were 
harvested by overlaying colonies with water and filtering off the suspension over $40 \mu \mathrm{m}$ cell strainers (VWR, Darmstadt, Germany). Liquid media were generally inoculated at a final concentration of $1 \times 10^{6}$ conidia per $\mathrm{ml}$. For the $\Delta$ areA strains, inoculation densities were generally doubled to compensate for reduced growth rates. For metabolite extraction and quantification liquid cultures in $50 \mathrm{ml}$ scale were used and incubated at $30^{\circ} \mathrm{C}$ for $48 \mathrm{hr}$ or $72 \mathrm{hr}$ depending on the specific experiment. For nitrogen or iron shift experiments, strains were pre-cultivated for $40 \mathrm{hr}$ in $150 \mathrm{ml}$ AMM-G100Gln50 or AMM-G100Gln50 with $200 \mu \mathrm{M} \mathrm{FeCl}$. Mycelia were harvested over sterile miracloth (Merck, Darmstadt, Germany) washed twice with AMM lacking a nitrogen (AMM-N) or iron source (AMM-Fe) and aliquots were transferred to fresh $50 \mathrm{ml}$ media with or without $50 \mathrm{mM} \mathrm{Gln}$ or with or without $200 \mu \mathrm{M} \mathrm{FeCl}_{3}$. Depending on the specific experiments, samples were analysed after 12, 24, or $48 \mathrm{hr}$ of cultivation.

\section{Metabolite extraction, quantification, purification, and structure elucidation}

Metabolites were extracted from culture broth as described previously (Gressler et alo, 2011). In brief, an equal volume of ethyl acetate was added and collected after defined shaking of the mixture. The procedure was repeated once. After evaporation of the solvent, residues were taken up in $1 \mathrm{ml}$ methanol each and filtered. Standard extract analyses were performed on an Agilent 1100 series HPLC-DAD system coupled with a MSD trap (Agilent Technologies, Waldbronn, Germany) operating in alternating ionisation mode. Terrein quantification was carried out from $50 \mathrm{ml}$ cultures as described elsewhere (Zaehle et al., 2014). For quantification of the siderophore coprogen, the complete $50 \mathrm{ml}$ culture supernatants were filtered and lyophilised to dryness. The remaining solids were extracted three times with $10 \mathrm{ml} \mathrm{MeOH}$. The solvent from the combined organic extracts was removed under reduced pressure and residues were redissolved in $2 \mathrm{ml} \mathrm{MeOH}$. The resulting slurries were filtered and the filtrates analysed by HPLC measurements. HPLC analyses were carried out on an Agilent 1260 device equipped with a quaternary pump and a UV/Nis detector (Agilent Technologies; Column: Zorbax Eclipse XDB-C8, $5 \mu \mathrm{m}, 150 \times 4.6 \mathrm{~mm}$; flow rate $1 \mathrm{ml} / \mathrm{min}$; eluent $\mathrm{A}: \mathrm{H}_{2} \mathrm{O} / 0.1 \% \mathrm{HCOOH}$, eluent $\mathrm{B}: \mathrm{MeOH}$ ). The gradient started with $10 \% \mathrm{~B}$ and reached $30 \% \mathrm{~B}$ after $4 \mathrm{~min}$, increased to $55 \% \mathrm{~B}$ within $10 \mathrm{~min}$ and reached $100 \% \mathrm{~B}$ after $2 \mathrm{~min}$, where it was retained for an additional $4 \mathrm{~min}$. Quantification of coprogen was performed from a calibration curve of known coprogen concentrations. For correlation of coprogen to the fungal biomass, mycelia from the cultures were dried for $48 \mathrm{hr}$ at $37^{\circ} \mathrm{C}$ and balanced and coprogen concentrations per gram dried mycelium were calculated. All quantifications were carried out in biological triplicates and technical duplicates. Isolation of coprogen for generation of the calibration curve was performed by semi-preparative HPLC from culture supernatants of the $\triangle a k u B$ and $\triangle a k u B \Delta t e r A$ strains and fractions were collected by automatic fraction collection. Separation was carried out on a Zorbax Eclipse XDB-C8, $5 \mu \mathrm{m}, 250 \times 4.6 \mathrm{~mm}$ with a flow rate of $4.0 \mathrm{ml} / \mathrm{min}$ using $\mathrm{H}_{2} \mathrm{O}$ as eluent $A$ and $\mathrm{MeOH}$ as eluent $B$. The gradient started with $10 \% \mathrm{~B}$, reached $30 \%$ B after $6.5 \mathrm{~min}$, increased to $55 \% \mathrm{~B}$ within $16.5 \mathrm{~min}$, reached $100 \% \mathrm{~B}$ after $2 \mathrm{~min}$, and was retained at $100 \% \mathrm{~B}$ for an additional $6 \mathrm{~min}$. For isolation of 2-((E)-prop-1-en-1-y))maleic acid, the crude product from upscaled terrein reduction assays (see below) was subjected to semi-preparative HPLC using a Zorbax Eclipse XDB-C8, $5 \mu \mathrm{m}, 250 \times 4.6 \mathrm{~mm}$ with a flow rate of $4.0 \mathrm{ml} / \mathrm{min}$, eluent $\mathrm{A}: \mathrm{H}_{2} \mathrm{O} / 0.1 \% \mathrm{HCOOH}$, eluent B: acetonitrile. The gradient started with $5 \% \mathrm{~B}$ and was held for $14 \mathrm{~min}$, increased to $10 \%$ B within 9 min, increased to $100 \%$ B within 2 min where it was retained for an additional 7 min. Fractions from the new metabolite formed from ferric iron reduction were collected and evaporated resulting in a white solid which revealed a $\mathrm{m} / z$ value of $150.0495\left[\mathrm{M}+\mathrm{H}^{+}\right]$by HRESI-MS that perfectly matched a calculated molecular formula of $\mathrm{C}_{7} \mathrm{H}_{8} \mathrm{O}_{4}$ containing four double-bond equivalents. ${ }^{13} \mathrm{C}-\mathrm{NMR}$ measurements (Figure 5-figure supplement 4) revealed the presence of two carbonyl groups, one terminal methyl group, and four carbons being part of a conjugated system. Two-dimensional NMR data (Figure 5-figure supplement 5) and analysis of all proton coupling constants from the ${ }^{1} \mathrm{H}-\mathrm{NMR}$ spectrum (Figure 5-figure supplement 4) finally confirmed the structure of 2-((E)-prop-1-en-1-yl)maleic acid. NMR spectra were recorded on a Bruker Avance III 500 and a Bruker Avance III 600 spectrometer (Bruker BioSpin GmbH, Rheinstetten, Germany) equipped with a cryoprobe head using DMSO-d6 and methanol-d4 as solvents and internal standards.

\section{Fruit infection and terrein quantification from fruits}

Fruit surfaces were wiped with a soft tissue saturated with $70 \%$ ethanol. Apples (ALDI, type: Tenroy Royal/Gala; Germany), bananas (type Bio, Fairverbindet; tegut, Jena, Germany), and nectarines 
(tegut, type: Sweet Lady, class 1, size A; Italy) were cut lengthwise using a sterile scalpel. The resulting groove was infected with $200 \mu$ of a conidia suspension containing $4 \times 10^{7}$ conidia and fruits were incubated for 7-10 $d$ at room temperature in a humid chamber. Cut but uninfected fruits served as controls. For cultivation in the presence of high levels of nitrogen, $200 \mu \mathrm{l}$ of a $3.5 \mathrm{M} \mathrm{NH}_{4} \mathrm{Cl}$ solution were applied prior to infection. For terrein quantification, fruits were homogenised and extracted twice with $100 \mathrm{ml}$ ethyl acetate and the solid residues were collected and evaporated for dry weight determination. Terrein was quantified from extracts as described above and terrein production rates were calculated as $\mathrm{mg}$ terrein/g fruit dry weight. A detailed description on fruit infection, sample preparation and terrein quantification is described in Bio-protocols by Gressler and Brock (2016).

\section{Fruit surface spot dilution assay}

To determine the induction of lesion formation on banana fruit surfaces, organic bananas (type Bio, Fairverbindet; tegut) were softly cleaned with water and air dried. $5 \mu$ of various sequential dilutions (1:2 to 1:512) of metabolite crude extracts (dissolved in PBS) were added as a single drop to the surface. Fruits were incubated in the dark at room temperature and photographs were taken after $40 \mathrm{hr}$ and $60 \mathrm{hr}$.

\section{Determination of ammonia in culture supernatants}

The determination of ammonia was performed as described by Weichselbaum et al. (1969). From each culture $1 \mathrm{ml}$ aliquots of broth were removed and centrifuged for $5 \mathrm{~min}$ at 16,000×g to remove residual mycelium. The supernatant was collected and adjusted to $\mathrm{pH} 7.0$ by addition of $\mathrm{NaOH}$. A $200 \mu \mathrm{l}$ aliquot was sequentially diluted in a nitrogen-free medium and transferred to a transparent flatbottom 96-well plate. After addition of $20 \mu \mathrm{l} \mathrm{SC}$ solution $\left(6.5 \mathrm{~g}\right.$ sodium salicylate $\left(\mathrm{C}_{7} \mathrm{H}_{5} \mathrm{O}_{3} \mathrm{Na}\right), 6.5 \mathrm{~g}$ trisodium citrate $\left(\mathrm{C}_{6} \mathrm{H}_{5} \mathrm{O}_{7} \mathrm{Na}_{3} \times 2 \mathrm{H}_{2} \mathrm{O}\right), 48.5 \mathrm{mg}$ disodium pentacyanonitrosylferrate $\left(\mathrm{Na}_{2} \mathrm{Fe}(\mathrm{CN})_{5} \mathrm{NO} \times\right.$ $2 \mathrm{H}_{2} \mathrm{O}$ ) in $50 \mathrm{ml}$ water), the reaction was started with $20 \mu \mathrm{l} \mathrm{DCIC}$ solution $(1.6 \mathrm{~g} \mathrm{NaOH}, 100 \mathrm{mg}$ sodium dichloroisocyanurate $\left(\mathrm{C}_{3} \mathrm{~N}_{3} \mathrm{Cl}_{2} \mathrm{O}_{3} \mathrm{Na}\right)$ in $50 \mathrm{ml}$ water). Readings were done after $4.5 \mathrm{hr}$ of incubation at room temperature in a FLOUstar Omega microplate reader (BMG Labtech, Ortenberg, Germany). Plates were shaken for $1 \mathrm{~min}$ in a double orbital direction and absorbance at $655 \mathrm{~nm}$ was measured (50 flashes/well, gain 2000). Fresh growth medium served as positive and water as negative controls. A calibration curve was recorded for calculation of ammonia levels from culture broth.

\section{Iron reduction assay}

The ferric iron-reducing assay was performed virtually as described by Benzie and Strain (1996). In brief, three different working solutions were prepared by mixing 20 parts of reagent $\mathrm{A}(0.4 \%$ sodium acetate, $1.6 \%$ acetic acid; $\mathrm{pH} 4)$ with 1 part of reagent B (0.4\% 2,4,6-tripyridyl-S-triazine (TPTZ; Sigma) in $0.16 \% \mathrm{HCl}$ and either 1 part of reagent $\mathrm{C} 1$ ( $88 \mathrm{mM}-5.5 \mathrm{mM}$ sequential dilutions of terrein), reagent C2 (2\% ascorbic acid, serving as positive control) or reagent C3 (water, serving as negative control). From each working solution, $200 \mu \mathrm{l}$ were transferred into wells of a 96-well plate. An iron stock solution $\left(10 \mathrm{mg} / \mathrm{ml} \mathrm{FeCl}_{3}\right.$ in $0.2 \mathrm{M} \mathrm{H}_{2} \mathrm{SO}_{4}$ ) was diluted to $0.05 \mathrm{mg} / \mathrm{ml}$ and $100 \mu \mathrm{l}$ were added to the working solution, which started the reaction. Reduction of ferric to ferrous iron was followed by determination of the change in absorbance at $590 \mathrm{~nm}$ for up to $10 \mathrm{hr}$ using a FLOUstar Omega microplate reader (BMG Labtech). For determination of the $\mathrm{pH}$ optimum of the terrein mediated reduction, reagent $\mathrm{A}$ was adjusted to $\mathrm{pH}$ values of 3.5-6.0. Reductive activity was normalised against the activity determined from ascorbic acid, which was set as $100 \%$. All experiments were carried out in triplicate. Upscaling of the iron reduction assays for metabolite extraction was performed by incubating $20 \mathrm{mg}(125 \mu \mathrm{mol})$ of terrein in a 1:2 molar ratio with either $\mathrm{FeSO}_{4}, \mathrm{FeCl}_{3}, \mathrm{CH}_{3} \mathrm{COOCu}$, or $\left(\mathrm{CH}_{3} \mathrm{COO}\right)_{2} \mathrm{Cu}$ in $10 \mathrm{ml}$ of reagent $\mathrm{A}$. Samples were incubated for $48 \mathrm{hr}$ at room temperature under continuous vertical rotation $(20 \mathrm{rpm})$. Metabolites were extracted three times with ethyl acetate $(10 \mathrm{ml}$ each). The solvent was evaporated and the residues dissolved in $\mathrm{MeOH}$ and analysed as described above.

\section{Chrome azol S (CAS) agar plate assays}

Chrome azol S agar plate assays were prepared as described by Milagres et al. (1999). Holes of $8 \mathrm{~mm}$ diameter were punched into plates and inoculated with $100 \mu \mathrm{l}$ methanolic solutions of coprogen, terrein, or propylene maleic acid in concentrations of $2.50,1.25,0.625$, and $0.3125 \mathrm{mg} / \mathrm{ml}$. Plates were incubated for $48 \mathrm{hr}$ at $30^{\circ} \mathrm{C}$ prior to photography. 


\section{RNA isolation, cDNA synthesis, and quantitative real-time PCR (qRT-PCR)}

Mycelia from specified cultivations were briefly washed with water and ground under liquid nitrogen. RNA was isolated using the RiboPure RNA Purification Kit (Ambion, Life Technologies, Darmstadt, Germany). Residual genomic DNA (gDNA) was removed by the DNA-free kit (Ambion). cDNA was synthesised by Revert Aid Reverse transcriptase (Thermo Scientific, Schwerte, Germany) using anchored oligodT primers. qRT-PCR was carried out on a CFX384 Touch Real-Time PCR Detection System (BioRad, Munich, Germany) using the EvaGreen $5 \times$ OPCR (ROX) Mix (Bio \& Sell, Feucht, Germany) following the manufacturer's protocol and using 1:5 and 1:10 dilutions of cDNA samples serving as templates. The actin gene (actA, ATEG_06973) and the enolase gene (enoA, ATEG_02902) were used for normalisation of transcript levels yielding similar results. Normalised transcript levels were calculated as fold expression $=2^{\Delta \text { (reference }- \text { target) }}$. Primers used for qRT-PCRs showed a primer efficiency of 1.89-2.0 and are listed in Figure 4-source data 2 (Figure 4-source data 2).

\section{Cloning strategies}

In general, all PCR amplifications were performed using the Phusion DNA Polymerase (Thermo Scientific). Plasmids were amplified in E. coli DH5 $\alpha$. A detailed description of all cloning procedures is given below. Oligonucleotides used for plasmid construction are listed in the Figure 4-source data 2 (Figure 4-source data 2). Fungal transformation was performed as described previously (Gressler et al., 2011) and transformants were checked by diagnostic PCR and Southern blot analyses (see Figure 4-figure supplement 4).

\section{Generation of PterA:lacZ reporter strains}

The promoter of the terA gene (PterA; $1220 \mathrm{bp}$ ) was amplified with oligonucleotides $\mathrm{P} 47 / 48$ from gDNA of $A$. terreus SBUG844 and ligated into the Notl/BamHI digested plasmid lacZ:trpCT-pJET1.2 (Gressler et al., 2011) containing the E. coli lacZ gene and the trpC terminator sequence. The plasmid was linearised by Notl digestion and the Notl-excised ptrA cassette from plasmid ptrA-pJET (Fleck and Brock, 2010) was inserted. The resulting plasmid was used for transformation of $A$. terreus SBUG844 wild-type resulting in SBUG844_PterA:lacZ.

\section{Overexpression of the atfA gene}

Constitutive strong expression of the atfA gene was performed by using the strong constitutive gpdA promoter from A. nidulans FGSC A4 (AnPgpdA, 1387 bp; oligonucleotides P49/50) to control expression of $A$. terreus atfA. The atfA ORF together with its natural terminator was amplified from gDNA of $A$. terreus SBUG844 (atfA + atfA ${ }^{\top}, 2466$ bp) using oligonucleotides P51/52. Both fragments were fused by in vitro recombination with the Spel- restricted hph-pCRIV vector using the InFusion HD cloning kit (Clonetech Laboratories, Saint-Germain-en-Laye, France). The resulting plasmid AnPgpdA:atfA:atfA ${ }^{\top}$ hph-pCRIV was used for transformation of $A$. terreus SBUG844 wild-type resulting in strain SBUG844_AnPgpdA:atfA.

\section{Deletion of the cpcA and rhbA gene}

The upstream and downstream fragments of cpcA (ATEG_03131) were amplified from gDNA of A. terreus SBUG844 using oligonucleotides P53/54 (943 bp) and P55/56 (853 bp). Similarly, the upstream and downstream flanks of rhbA (ATEG_09480) were amplified with oligonucleotides $\mathrm{P} 57 / 58$ (473 bp) and P59/60 (418 bp). The respective fragments were fused by in vitro recombination with the ptrA resistance cassette (1950 bp) from ptrA-pJET1 (Fleck and Brock, 2010) into the Kpnl-restricted pUC19 vector using the InFusion HD cloning kit (Clonetech Laboratories), resulting in cpcAup-ptrAcpcAdn-pUC19, rhbAup-ptrA-rhbAdn-pUC19, and dnmTup-ptrA-dnmTdn-pUC19. The deletion cassettes were excised by Kpnl restriction and used for transformation of SBUG844 $\Delta a k u B$.

\section{Deletion and complementation of the areA gene}

The upstream and downstream flanks of areA (ATEG_07264) were amplified from gDNA of $A$. terreus SBUG844 with oligonucleotides P61/62 (820 bp) and P63/64 (731 bp). Fragments were fused by fusion PCR and ligated into the pJET1.2 cloning vector (Thermo Scientific). The fragment was excised with Smal and subcloned into pUC19. After restriction with Notl, the ptrA resistance cassette (1950 bp) from ptrA-pJET1 (Fleck and Brock, 2010) was inserted resulting in areAup-ptrA-areAdn-pUC19. 
The deletion cassette was excised by Smal and used for transformation of SBUG844 $\Delta a k u B$. For complementation, a fragment spanning the entire areA ORF including its promoter and terminator sequence was amplified from gDNA of the $A$. terreus type strain FGSC A1156 (NIH2624) using oligonucleotides P65/64 (4181 bp) and ligated into pJET1.2. gDNA from the type strain was used because its areA promoter sequence contained an additional Pstl restriction site that allowed discrimination of complemented strains from the parental SBUG844 $\triangle a k u B$ strain. The complementation fragment was excised by Xhol restriction from plasmid A1156(areAup:areA:areAdn)-pJET1.2 and directly used for the transformation of SBUG844 $\Delta a k u B \Delta a r e A$. Due to the inability of the $\Delta$ areA mutant to utilise various nitrogen sources, no additional resistance marker was required and transformants were regenerated on media containing nitrate as the sole nitrogen source. Complemented strains were checked by Southern blot analysis and an additional Pstl control digest of the PCR-amplified areA upstream flank.

\section{Deletion and complementation of the atfA gene}

The upstream and downstream fragments of atfA (ATEG_04664) were amplified from gDNA of A. terreus SBUG844 with oligonucleotides P66/67 (1102 bp) and P68/69 (890 bp) and fused by in vitro recombination with the Notl-excised ptrA resistance cassette (1950 bp) from ptrA-pJET1 (Fleck and Brock, 2010) into the Kpnl-excised pUC19 vector using the InFusion HD cloning kit (Clonetech Laboratories) resulting in atfAup-ptrA-atfAdn-pUC19. The deletion cassette was excised by Kpnl and used for transformation of SBUG844 $\triangle a k u B$. For deletion of atfA in the $\triangle$ areA background, the ptrA resistance cassette was replaced by the phleomycin resistance (ble) cassette (Notl digest from ble-pJET1.2). The deletion cassette was excised by Kpnl restriction and used for transformation of SBUG844 $\triangle a k u B \Delta a r e A$. For complementation of the $\triangle a t f A$ phenotype, a fragment spanning the entire atfA gene including its promoter and terminator region were amplified with oligonucleotides P66/70 (3319 bp) and an additional downstream fragment was amplified with P71/69 (859 bp). The fragments were fused by in vitro recombination with the Notl-excised ble resistance cassette (2073 bp) from ble-pJET1.2 into the Kpnl-restricted pUC19 vector using the InFusion HD cloning kit (Clonetech Laboratories) resulting in atfAup:atfA:atfA ${ }^{\top} \_b l e \_a t f A d n-p U C 19$. The complementation cassette was excised by Kpnl and used for transformation of SBUG844 $\Delta a k u B \Delta a t f A$.

\section{Overexpression of terR in the $\Delta$ areA/ $\Delta$ atfA background}

The atf $A$ flanking regions were used to integrate the ter $R$ gene under control of the $A$. nidulans gpd $A$ promoter in the atfA locus, which results in an atfA deletion. The upstream and downstream flanks of atfA were amplified from gDNA of $A$. terreus SBUG844 using oligonucleotides P72/73 (1125 bp) and P74/75 (913 bp). Subsequently, a fragment already containing the fusion of AnPgpdA with the terR ORF including its native terminator (4405 bp) was amplified with oligonucleotides P76/77 from plasmid hph_AnPgpdA:terR-pUC19 (Gressler et al., 2015a). The plasmid hph-pCRIV (Fleck and Brock, 2010) was restricted with EcoRl to remove the hph resistance cassette and all three fragments were fused by in vitro recombination into the EcoRl site using the InFusion HD cloning kit (Clonetech Laboratories). The resulting plasmid atfAup_AnPgpdA:terR:terR__atfAdn_pCRIV was linearised with Notl and the ble resistance cassette from ble-pJET1.2 was inserted between the terR terminator and the atfA downstream region. The final plasmid was restricted with EcoRl, and the fragment atfAup_AnPgpdA:terR: terR $R^{\top}$ ble_atfAdn was used for transformation of $A$. terreus SBUG844 $\triangle a k u B \Delta$ areA to replace the atfA ORF with the terR overexpression construct.

\section{Partial deletion of hapX, complementation with AnhapX, and hapX sequence verification}

Because the complete sequence information of the hapX locus was lacking at the beginning of this study, only a partial deletion of the $A$. terreus hapX gene was performed. Upstream and downstream flanks inside the hapX coding region (ATEG_08014) were amplified with oligonucleotides P82/83 (387 bp) and P84/85 (345 bp) from genomic DNA of SBUG844 and fused by in vitro recombination with the ptrA resistance cassette (1950 bp) from ptrA-pJET1 (Fleck and Brock, 2010) into the Kpnl-restricted pUC19 vector using the InFusion HD cloning kit (Clonetech Laboratories) resulting in hapXup-ptrAhapXdn-pUC19. The deletion construct was excised by Kpnl restriction and used for transformation of SBUG844 $\Delta a k u B$. For complementation of the partial hapX deletion by the $A$. nidulans hapX, the 
upstream and downstream flanks of ATEG_08014 used for generation of the partial deletion construct were amplified with oligonucleotides P82/86 (381 bp) and P87/85 (341 bp) and the complete hapX ORF (AN8251) including its native promoter and terminator sequence was amplified from gDNA of A. nidulans FGSC A4 using oligonucleotides P88/89 (2648 bp). All fragments were fused by in vitro recombination with the ble resistance cassette (2073 bp) from ble-pJET1.2 (2073 bp) into the Kpnlrestricted pUC19 vector using the InFusion HD cloning kit (Clonetech Laboratories) resulting in hapXup_PAnhapX:AnhapX:AnhapX_ble_hapXdn-pUC19. The complementation cassette was excised by Kpnl and used for transformation of SBUG844 $\Delta a k u B \Delta$ hapX. To identify the complete coding region of the $A$. terreus hapX gene, long run PCRs from within the hapX gene and the last nucleotides of the known 5'- and 3'- borders were performed with oligonucleotides P110/111 (800 bp 5' fragment) and P112/113 (2500 bp 3' fragment). Bands were excised from agarose gels and cloned into the pJET1.2 cloning vector. Fragments were sequenced from both strands using the primer walking method (oligonucleotides P114/115 for both fragments and additionally P116, P117 and P78 for the 3' fragment). Finally, RNA was isolated from iron starvation conditions, transcribed into cDNA, and the hapX ORF was amplified with gene-specific oligonucleotides $113 / 79$ and sequenced with oligonucleotides P114/115. The complete hapX locus information was submitted to EMBL and can be found under accession number KP233834(Gressler et alo, 2015b).

\section{Deletion and complementation of the sreA gene}

The upstream and downstream flanking regions of sreA (ATEG_07714) were amplified from gDNA of A. terreus SBUG844 with oligonucleotides P90/91 (763 bp) and P92/93 (756 bp) and fused by in vitro recombination with the ptrA resistance cassette (1950 bp) from ptrA-pJET1 (Fleck and Brock, 2010) into the Kpnl-restricted pUC19 vector using the InFusion HD cloning kit (Clonetech Laboratories) resulting in sreAup-ptrA-sreAdn-pUC19. The deletion cassette was excised by Kpnl and used for transformation of SBUG844 $\triangle a k u B$. For complementation, a fragment spanning the sreA coding region including its natural promoter and terminator sequence was amplified with oligonucleotides P94/95 (3018 bp) and additional downstream fragment with P96/97 (970 bp). The fragments were fused by in vitro recombination with the ble resistance cassette (2073 bp) from ble-pJET1.2 into the Kpnl-restricted pUC19 vector using the InFusion HD cloning kit (Clonetech Laboratories) resulting in sreAup:sreA:sreA $A^{\top} \_b l e \_s r e A d n-p U C 19$. The complementation cassette was excised by Kpnl restriction and used for transformation of SBUG844 $\Delta a k u B \Delta s r e A$.

\section{Deletion and complementation of the sidA gene}

The upstream and downstream fragments of the sidA gene (ATEG_06879) were amplified from gDNA of A. terreus SBUG844 using oligonucleotides P98/99 (764 bp) and P100/101 (939 bp) and fused by in vitro recombination with the ptrA resistance cassette (1950 bp) from ptrA-pJET1 (Fleck and Brock, 2010) into the Kpnl-restricted pUC19 vector using the InFusion HD cloning kit (Clonetech Laboratories) resulting in sidAup-ptrA-sidAdn-pUC19. For transformation of the terA deletion mutant, the ptrA cassette was excised by Notl restriction and replaced by the ble cassette. The deletion cassette was excised by Kpnl restriction and used for transformation of SBUG844 $\Delta a k u B$ and SBUG844 $\Delta a k u B \Delta t e r A$. For complementation of strain SBUG844 $\Delta$ akuB $\Delta$ sidA, the sidA ORF including its promoter and terminator sequence was amplified with oligonucleotides P102/103 (2393 bp) and an additional downstream fragment was amplified with P104/ 105 (919 bp). The fragments were fused by in vitro recombination with the ble resistance cassette (2073 bp) from ble-pJET1.2 into the Hindll-restricted pUC19 vector using the InFusion HD cloning kit (Clonetech Laboratories) resulting in sidAup:sidA:sidA ${ }^{\top} \_b l e \_s i d A d n-p U C 19$. The complementation cassette was excised by Hindlll restriction and used for transformation of SBUG844 $\Delta a k u B \Delta \operatorname{sid} A$.

\section{Bacterial expression and purification of AreA polypeptide for SPR analysis}

The coding sequence for the A. nidulans AreA DNA-binding domain (amino acids 663-797) was amplified from plasmid pGEX4T1-AreA-ZnF (Muro-Pastor et al., 2004) as BamHI-HindIII fragment using oligonucleotides P80/81 (Figure 4-source data 2). The amplified fragment was cloned into a modified pET-43.1a vector allowing the addition of a $\mathrm{N}$-terminal ( $\mathrm{His}_{6}$-tag and removable by a tobacco etch virus (TEV) protease (Novagen). (His) 6 -AreA $663-797$ was produced by E. coli BL21 (DE3) cells grown at $30^{\circ} \mathrm{C}$ in 1 I Overnight Express Instant TB Medium (Novagen, Darmstadt, Germany) in the presence of $1 \mathrm{mM} \mathrm{Zn}(\mathrm{OAc})_{2}$. Cells (15-20 $\mathrm{g}$ wet weight) were collected by centrifugation, resuspended in $200 \mathrm{ml}$ lysis buffer $\left(50 \mathrm{mM} \mathrm{NaH}{ }_{2} \mathrm{PO}_{4}, 300 \mathrm{mM} \mathrm{NaCl}, 10 \%\right.$ vol/vol glycerol, $40 \mathrm{mM}$ imidazole, $5 \mathrm{mM}$ 
$\beta$-mercaptoethanol, $1 \mathrm{mM}$ AEBSF, $\mathrm{pH}$ 8.0) and disrupted using an Emulsiflex C5 high pressure homogeniser (Avestin, Mannheim, Germany). Cleared cellular extract was loaded to a $25 \mathrm{ml} \mathrm{Ni}$ Sepharose FF (GE Heathcare, Freiburg, Germany) column and (His) -AreA $_{663-797}$ was eluted with $200 \mathrm{mM}$ imidazole. After removal of the $(\mathrm{His})_{6}$ tag by adding $4 \mu \mathrm{g}$ TEV protease per mg peptide and overnight incubation at room temperature, samples were adjusted to $150 \mathrm{mM} \mathrm{NaCl}$ and applied on a $40 \mathrm{ml}$ CellufineSulfate (Millipore, Darmstatdt, Germany) column that was equilibrated with $50 \mathrm{mM} \mathrm{NaH}_{2} \mathrm{PO}_{4}, 150 \mathrm{mM} \mathrm{NaCl}, 10 \%$ vol/vol glycerol, $5 \mathrm{mM} \beta$-mercaptoethanol, $10 \mu \mathrm{M} \mathrm{Zn(OAc)} 2, \mathrm{pH} 7.5$, followed by elution of AreA $663-797$ with a gradient up to $2 \mathrm{M} \mathrm{NaCl}$. Peak fractions were concentrated with an Amicon Ultra-15 10K centrifugal filter device and purified to homogeneity by size exclusion chromatography on a Superdex 75 prep grade column (GE Healthcare) in 20 mM HEPES, 300 mM NaCl, 5 mM $\beta$-mercaptoethanol, $10 \mu \mathrm{M} \mathrm{Zn(OAc)} 2, \mathrm{pH}$ 7.5 as running buffer. The absolute molecular mass of $A_{r e} A_{663-797}$ was determined in series on a miniDawn TREOS static light scattering monitor and an Optilab T-rEX differential refractometer (Wyatt, Dernbach, Germany). The molar mass was calculated using ASTRA 6 software (Wyatt). AreA $663-797$ was stored in 50\% $\mathrm{vol} / \mathrm{vol}$ glycerol at $-20^{\circ} \mathrm{C}$.

\section{SPR measurements}

Real-time analyses were performed on a Biacore 2000 system (GE Healthcare) at $25^{\circ} \mathrm{C}$. DNA duplexes were produced by annealing complementary oligonucleotides using a fivefold molar excess of the non-biotinylated oligonucleotide. The dsDNA was injected on flow cells of a streptavidin (Sigma)coated CM3 sensor chip at a flow rate of $10 \mu \mathrm{l} / \mathrm{min}$ until the calculated amount of DNA that gives a maximum $A_{r e} A_{663-797}$ binding capacity of $100 \mathrm{RU}$ were bound. AreA $663-797$ was injected in running buffer (10 mM HEPES pH 7.4, $150 \mathrm{mM} \mathrm{NaCl}, 0.005 \%$ (vol/vol) surfactant P20, $5 \mathrm{mM}$ $\beta$-mercaptoethanol and $1 \mu \mathrm{M} \mathrm{ZnCl}_{2}$ ) at concentrations from 3.125 to $200 \mathrm{nM}$. Sample injection and dissociation times were set to 200 and $400 \mathrm{~s}$ at a flow rate of $30 \mu \mathrm{l} / \mathrm{min}$. Regeneration was achieved with $10 \mathrm{mM}$ Tris/ $\mathrm{HCl} \mathrm{pH} \mathrm{7.5,0.5} \mathrm{M} \mathrm{NaCl} 1 \mathrm{mM}$ EDTA and 0.005\% (wt/vol) SDS for $1 \mathrm{~min}$. Refractive index errors due to bulk solvent effects were corrected with responses from DNA-free flow cell 1 as well as subtracting blank injections. Kinetic raw data were processed and globally fitted with Scrubber 2.0c (BioLogic Software) using a 1:1 interaction model including a mass transport term.

\section{Statistical significance}

All analyses in which statistical analyses were required were performed in biological triplicates with at least two technical replications. Significance was calculated by use of the Microsoft Excel 2007 software package using the Student's paired t-test with a two-tailed distribution; $p$ values were marked as follows: ${ }^{\star} \mathrm{p}<0.05,{ }^{* \star} \mathrm{p}<0.01 ;{ }^{* \star *} \mathrm{p}<0.001$.

\section{Acknowledgements}

We greatly acknowledge Daniela Hildebrandt for assistance in plasmid construction, Carmen Karkowski and Elena Geib for assistance in metabolite quantification, Andrea Perner for HR-MS analyses, and Heike Heinecke for recording ${ }^{1} \mathrm{H}$ - and ${ }^{13} \mathrm{C}$-NMR spectra of secondary metabolites. This work was supported by the German Science Foundation (DFG, BR-2216/4-1) and internal funding from the Hans Knoell Institute.

\section{Additional information}

\begin{tabular}{|c|c|c|}
\hline Funder & Grant reference & Author \\
\hline $\begin{array}{l}\text { Deutsche } \\
\text { Forschungsgemeinschaft } \\
\text { (DFG) }\end{array}$ & BR-2216/4-1 & $\begin{array}{l}\text { Markus Gressler, Matthias } \\
\text { Brock }\end{array}$ \\
\hline Hans Knöll Institute & internal funding & $\begin{array}{l}\text { Markus Gressler, Florian } \\
\text { Meyer, Daniel Heine, Peter } \\
\text { Hortschansky, Christian } \\
\text { Hertweck, Matthias Brock }\end{array}$ \\
\hline
\end{tabular}

The funders had no role in study design, data collection and interpretation, or the decision to submit the work for publication. 
Author contributions

MG, Conception and design, Acquisition of data, Analysis and interpretation of data, Drafting or revising the article; FM, DH, $\mathrm{PH}$, Acquisition of data, Analysis and interpretation of data, Drafting or revising the article; $\mathrm{CH}$, Analysis and interpretation of data, Drafting or revising the article; $\mathrm{MB}$, Conception and design, Analysis and interpretation of data, Drafting or revising the article

\section{Additional files}

Major dataset

The following dataset was generated:

\begin{tabular}{|c|c|c|c|c|}
\hline Author(s) & Year & Dataset title & $\begin{array}{l}\text { Dataset ID } \\
\text { and/or URL }\end{array}$ & $\begin{array}{l}\text { Database, license, and } \\
\text { accessibility information }\end{array}$ \\
\hline $\begin{array}{l}\text { Gressler M, Meyer F, } \\
\text { Heine D, Hortschansky P, } \\
\text { Brock M }\end{array}$ & 2015 & $\begin{array}{l}\text { Aspergillus terreus HapX } \\
\text { bZIP transcriptional iron } \\
\text { regulator (hapX) gene, } \\
\text { promoter region and } \\
\text { complete cds }\end{array}$ & $\begin{array}{l}\text { http://www.ebi.ac.uk/ } \\
\text { ena/data/view/KP233834 }\end{array}$ & $\begin{array}{l}\text { Publicly available at the } \\
\text { European Nucleotide } \\
\text { Archive (accession no. } \\
\text { KP233834). }\end{array}$ \\
\hline
\end{tabular}

\section{References}

Arst HN Jr, Cove DJ. 1973. Nitrogen metabolite repression in Aspergillus nidulans. Molecular and General Genetics 126:111-141. doi: 10.1007/BF00330988.

Baddley JW, Pappas PG, Smith AC, Moser SA. 2003. Epidemiology of Aspergillus terreus at a university hospital. Journal of Clinical Microbiology 41:5525-5529. doi: 10.1128/JCM.41.12.5525-5529.2003.

Balazs A, Pocsi I, Hamari Z, Leiter E, Emri T, Miskei M, Olah J, Toth V, Hegedus N, Prade RA, Molnar M, Pocsi I. 2010. AtfA bZIP-type transcription factor regulates oxidative and osmotic stress responses in Aspergillus nidulans. Molecular Genetics and Genomics 283:289-303. doi: 10.1007/s00438-010-0513-z.

Benzie IF, Strain JJ. 1996. The ferric reducing ability of plasma (FRAP) as a measure of "antioxidant power": the FRAP assay. Analytical Biochemistry 239:70-76. doi: 10.1006/abio.1996.0292.

Bertrand S, Bouchara JP, Venier MC, Richomme P, Duval O, Larcher G. 2010. N(alpha)-methyl coprogen B, a potential marker of the airway colonization by Scedosporium apiospermum in patients with cystic fibrosis. Medical Mycology 48(Suppl 1):S98-S107. doi: 10.3109/13693786.2010.503972.

Bhatnagar D, Cary JW, Ehrlich K, Yu J, Cleveland TE. 2006. Understanding the genetics of regulation of aflatoxin production and Aspergillus flavus development. Mycopathologia 162:155-166. doi: 10.1007/s11046-006-0050-9.

Bizukojc M, Ledakowicz S. 2015. Bioprocess Engineering aspects of the cultivation of a lovastatin producer Aspergillus terreus. Advances in Biochemical Engineering/Biotechnology 149:133-170. doi: 10.1007/ 10_2014_302.

Blatzer M, Barker BM, Willger SD, Beckmann N, Blosser SJ, Cornish EJ, Mazurie A, Grahl N, Haas H, Cramer RA. 2011. SREBP coordinates iron and ergosterol homeostasis to mediate triazole drug and hypoxia responses in the human fungal pathogen Aspergillus fumigatus. PLOS Genetics 7:e1002374. doi: 10.1371/journal.pgen.1002374.

Boukhalfa H, Crumbliss AL. 2002. Chemical aspects of siderophore mediated iron transport. Biometals 15: 325-339. doi: 10.1023/A:1020218608266.

Brakhage AA, Spröte P, Al-Abdallah Q, Gehrke A, Plattner H, Tüncher A. 2004. Regulation of penicillin biosynthesis in filamentous fungi. Advances in Biochemical Engineering/Biotechnology 88:45-90. doi: 10.1007/ b99257.

Chang PK, Ehrlich KC, Linz JE, Bhatnagar D, Cleveland TE, Bennett JW. 1996. Characterization of the Aspergillus parasiticus niaD and niiA gene cluster. Current Genetics 30:68-75. doi: 10.1007/s002940050102.

Chang PK, Yu J, Bhatnagar D, Cleveland TE. 2000. Characterization of the Aspergillus parasiticus major nitrogen regulatory gene, areA. Biochimica et Biophysica Acta 1491:263-266. doi: 10.1016/S0167-4781(00)00004-X.

Churchland C, Grayston SJ. 2014. Specificity of plant-microbe interactions in the tree mycorrhizosphere biome and consequences for soil C cycling. Frontiers in Microbiology 5:261. doi: 10.3389/fmicb.2014.00261.

Dakora FD, Phillips DA. 2002. Root exudates as mediators of mineral acquisition in low-nutrient environments. Plant and Soil 245:35-47. doi: 10.1023/A:1020809400075.

Davis MA, Askin MC, Hynes MJ. 2005. Amino acid catabolism by an areA-regulated gene encoding an L-amino acid oxidase with broad substrate specificity in Aspergillus nidulans. Applied and Environmental Microbiology 71: 3551-3555. doi: 10.1128/AEM.71.7.3551-3555.2005.

Eisendle M, Oberegger H, Zadra I, Haas H. 2003. The siderophore system is essential for viability of Aspergillus nidulans: functional analysis of two genes encoding L-ornithine N5-monooxygenase (sidA) and a non-ribosomal peptide synthetase (sidC). Molecular Microbiology 49:359-375. doi: 10.1046/j.1365-2958.2003.03586.x.

Elmagirbi A, Sulistyarti H, Atikah. 2012. Study of ascorbic acid as iron(III) reducing agent for spectrophotometric iron speciation. The Journal of Pure and Applied Chemistry Research 1:11-17.

Fleck CB, Brock M. 2010. Aspergillus fumigatus catalytic glucokinase and hexokinase: expression analysis and importance for germination, growth, and conidiation. Eukaryotic Cell 9:1120-1135. doi: 10.1128/EC.00362-09. 
Gao H, Guo W, Wang Q, Zhang L, Zhu M, Zhu T, Gu Q, Wang W, Li D. 2013. Aspulvinones from a mangrove rhizosphere soil-derived fungus Aspergillus terreus Gwq-48 with anti-influenza A viral (H1N1) activity. Bioorganic \& Medicinal Chemistry Letters 23:1776-1778. doi: 10.1016/j.bmcl.2013.01.051.

Giese H, Sondergaard TE, Sorensen JL. 2013. The AreA transcription factor in Fusarium graminearum regulates the use of some nonpreferred nitrogen sources and secondary metabolite production. Fungal Biology 117:814-821. doi: 10.1016/j.funbio.2013.10.006.

Gressler M, Brock M. 2016. Aspergillus terreus infection of fruits and terrein quantification by HPLC analysis. Bio-protocol 6:e1845. doi: 10.21769/BioProtoc.1845.

Gressler M, Hortschansky P, Geib E, Brock M. 2015a. A new high-performance heterologous fungal expression system based on regulatory elements from the Aspergillus terreus terrein gene cluster. Frontiers in Microbiology 6:184. doi: 10.3389/fmicb.2015.00184.

Gressler M, Meyer F, Heine D, Hortschansky P, Brock M. 2015b. Aspergillus terreus HapX bZIP transcriptional iron regulator (hapX) gene, promoter region and complete cds. European Nucleotide Archive KP233834. http://www. ebi.ac.uk/ena/data/view/KP233834.

Gressler M, Zaehle C, Scherlach K, Hertweck C, Brock M. 2011. Multifactorial induction of an orphan PKS-NRPS gene cluster in Aspergillus terreus. Chemistry \& Biology 18:198-209. doi: 10.1016/j.chembiol. 2010.12.011.

Gsaller F, Hortschansky P, Beattie SR, Klammer V, Tuppatsch K, Lechner BE, Rietzschel N, Werner ER, Vogan AA, Chung D, Muhlenhoff U, Kato M, Cramer RA, Brakhage AA, Haas H. 2014. The Janus transcription factor HapX controls fungal adaptation to both iron starvation and iron excess. The EMBO Journal 33:2261-2276. doi: 10. 15252/embj.201489468.

Haas H. 2012. Iron-a key nexus in the virulence of Aspergillus fumigatus. Frontiers in Microbiology 3:28. doi: 10. 3389/fmicb.2012.00028.

Haas H. 2014. Fungal siderophore metabolism with a focus on Aspergillus fumigatus. Natural Product Reports 31: 1266-1276. doi: 10.1039/C4NP00071D.

Hider RC, Kong X. 2010. Chemistry and biology of siderophores. Natural Product Reports 27:637-657. doi: 10. 1039/b906679a.

Hoffmann B, Valerius O, Andermann M, Braus GH. 2001. Transcriptional autoregulation and inhibition of mRNA translation of amino acid regulator gene cpcA of filamentous fungus Aspergillus nidulans. Molecular Biology of the Cell 12:2846-2857. doi: 10.1091/mbc.12.9.2846.

Hong SY, Roze LV, Linz JE. 2013. Oxidative stress-related transcription factors in the regulation of secondary metabolism. Toxins 5:683-702. doi: 10.3390/toxins5040683.

Hortschansky P, Eisendle M, Al-Abdallah Q, Schmidt AD, Bergmann S, Thon M, Kniemeyer O, Abt B, Seeber B, Werner ER, Kato M, Brakhage AA, Haas H. 2007. Interaction of HapX with the CCAAT-binding complex-a novel mechanism of gene regulation by iron. The EMBO Journal 26:3157-3168. doi: 10.1038/ sj.emboj.7601752.

Hortschansky P, Ando E, Tuppatsch K, Arikawa H, Kobayashi T, Kato M, Haas H, Brakhage AA. 2015. Deciphering the combinatorial DNA-binding code of the CCAAT-binding complex and the iron-regulatory basic region leucine zipper (bZIP) transcription factor HapX. The Journal of Biological Chemistry 290:6058-6070. doi: 10. 1074/jbc.M114.628677.

Howard DH, Rafie R, Tiwari A, Faull KF. 2000. Hydroxamate siderophores of Histoplasma capsulatum. Infection and Immunity 68:2338-2343. doi: 10.1128/IAI.68.4.2338-2343.2000.

Hunter CC, Siebert KS, Downes DJ, Wong KH, Kreutzberger SD, Fraser JA, Clarke DF, Hynes MJ, Davis MA, Todd RB. 2014. Multiple nuclear localization signals mediate nuclear localization of the GATA transcription factor AreA. Eukaryotic Cell 13:527-538. doi: 10.1128/EC.00040-14.

Hynes MJ. 1975. Studies on the role of the areA gene in the regulation of nitrogen catabolism in Aspergillus nidulans. Australian Journal of Biological Sciences 28:301-313.

Johnstone IL, Mccabe PC, Greaves P, Gurr SJ, Cole GE, Brow MA, Unkles SE, Clutterbuck AJ, Kinghorn JR, Innis MA. 1990. Isolation and characterisation of the crnA-niiA-niaD gene cluster for nitrate assimilation in Aspergillus nidulans. Gene 90:181-192. doi: 10.1016/0378-1119(90)90178-T.

Kamata M, Sakai H, Hirota A. 1983. Isolation of acetyl aranotin bis dethiodimethylthioacetyl aranotin and terrein as plant growth inhibitors from a strain of Aspergillus terreus. Agricultural and Biological Chemistry 47:2637-2638. doi: 10.1271/bbb1961.47.2637.

Kim H, Woloshuk CP. 2008. Role of AREA, a regulator of nitrogen metabolism, during colonization of maize kernels and fumonisin biosynthesis in Fusarium verticillioides. Fungal Genetics and Biology 45:947-953. doi: 10.1016/j. fgb.2008.03.007.

Krappmann S, Bignell EM, Reichard U, Rogers T, Haynes K, Braus GH. 2004. The Aspergillus fumigatus transcriptional activator $\mathrm{CpcA}$ contributes significantly to the virulence of this fungal pathogen. Molecular Microbiology 52:785-799. doi: 10.1111/j.1365-2958.2004.04015.x.

Kreutzer MF, Nett M. 2012. Genomics-driven discovery of taiwachelin, a lipopeptide siderophore from Cupriavidus taiwanensis. Organic \& Biomolecular Chemistry 10:9338-9343. doi: 10.1039/c2ob26296g.

Lara-Rojas F, Sanchez O, Kawasaki L, Aguirre J. 2011. Aspergillus nidulans transcription factor AtfA interacts with the MAPK SakA to regulate general stress responses, development and spore functions. Molecular Microbiology 80:436-454. doi: 10.1111/j.1365-2958.2011.07581.x.

Lass-Flörl C, Griff K, Mayr A, Petzer A, Gastl G, Bonatti H, Freund M, Kropshofer G, Dierich MP, Nachbaur D. 2005. Epidemiology and outcome of infections due to Aspergillus terreus: 10-year single centre experience. British Journal of Haematology 131:201-207. doi: 10.1111/j.1365-2141.2005.05763.x. 
Louis B, Roy P, Sayanika D, Talukdar N. 2013. Aspergillus terreus Thom a new pathogen that causes foliar blight of potato. Plant Pathology \& Quarantine 3:29-33. doi: 10.5943/ppq/3/1/5.

Louis B, Waikhom SD, Roy P, Bhardwaj PK, Singh MW, Chandradev SK, Talukdar NC. 2014a. Invasion of Solanum tuberosum L. by Aspergillus terreus: a microscopic and proteomics insight on pathogenicity. BMC Research Notes 7:350. doi: 10.1186/1756-0500-7-350.

Louis B, Waikhom SD, Singh WM, Talukdar NC, Roy P. 2014b. Diversity of ascomycetes at the potato interface: new devastating fungal pathogens posing threat to potato farming. Plant Pathology Journal 13:18-27. doi: 10. 3923/ppj.2014.18.27.

Melo IS, Faull JL, Nascimento RS. 2006. Antagonism of Aspergillus terreus to Sclerotinia sclerotiorum. Brazilian Journal of Microbiology 37:417-419. doi: 10.1590/S1517-83822006000400002.

Mihlan M, Homann V, Liu TW, Tudzynski B. 2003. AREA directly mediates nitrogen regulation of gibberellin biosynthesis in Gibberella fujikuroi, but its activity is not affected by NMR. Molecular Microbiology 47:975-991. doi: 10.1046/j.1365-2958.2003.03326.x.

Milagres AM, Machuca A, Napoleao D. 1999. Detection of siderophore production from several fungi and bacteria by a modification of chrome azurol S (CAS) agar plate assay. Journal of Microbiological Methods 37:1-6. doi: 10. 1016/S0167-7012(99)00028-7.

Muro-Pastor MI, Strauss J, Ramon A, Scazzocchio C. 2004. A paradoxical mutant GATA factor. Eukaryotic Cell 3: 393-405. doi: 10.1128/EC.3.2.393-405.2004.

Nimmanee P, Woo PC, Vanittanakom P, Youngchim S, Vanittanakom N. 2014. Functional analysis of atfA gene to stress response in pathogenic thermal dimorphic fungus Penicillium marneffei. PLOS ONE 9:e111200. doi: 10. 1371/journal.pone.0111200.

Panepinto JC, Oliver BG, Amlung TW, Askew DS, Rhodes JC. 2002. Expression of the Aspergillus fumigatus rheb homologue, rhbA, is induced by nitrogen starvation. Fungal Genetics and Biology 36:207-214. doi: 10.1016/ S1087-1845(02)00022-1.

Pollack JR, Neilands JB. 1970. Enterobactin, an iron transport compound from Salmonella typhimurium. Biochemical and Biophysical Research Communications 38:989-992. doi: 10.1016/0006-291X(70)90819-3.

Proft M, Gibbons FD, Copeland M, Roth FP, Struhl K. 2005. Genomewide identification of Sko1 target promoters reveals a regulatory network that operates in response to osmotic stress in Saccharomyces cerevisiae. Eukaryotic Cell 4:1343-1352. doi: 10.1128/EC.4.8.1343-1352.2005.

Punt PJ, Strauss J, Smit R, Kinghorn JR, Van Den Hondel CA, Scazzocchio C. 1995. The intergenic region between the divergently transcribed niiA and niaD genes of Aspergillus nidulans contains multiple NirA binding sites which act bidirectionally. Molecular and Cellular Biology 15:5688-5699.

Rajalakshmi S, Mahesh N. 2014. Production and characterization of bioactive metabolites isolated from Aspergillus terreus in rhizosphere soil of medicinal plants. International Journal of Current Microbiology and Applied Sciences 3:784-798.

Ravagnani A, Gorfinkiel L, Langdon T, Diallinas G, Adjadj E, Demais S, Gorton D, Arst HN Jr, Scazzocchio C. 1997. Subtle hydrophobic interactions between the seventh residue of the zinc finger loop and the first base of an HGATAR sequence determine promoter-specific recognition by the Aspergillus nidulans GATA factor AreA. The EMBO Journal 16:3974-3986. doi: 10.1093/emboj/16.13.3974.

Rohlfs M, Churchill AC. 2011. Fungal secondary metabolites as modulators of interactions with insects and other arthropods. Fungal Genetics and Biology 48:23-34. doi: 10.1016/j.fgb.2010.08.008.

Roje S. 2006. S-Adenosyl-L-methionine: beyond the universal methyl group donor. Phytochemistry 67:1686-1698. doi: 10.1016/j.phytochem.2006.04.019.

Schrettl M, Beckmann N, Varga J, Heinekamp T, Jacobsen ID, Jochl C, Moussa TA, Wang S, Gsaller F, Blatzer M, Werner ER, Niermann WC, Brakhage AA, Haas H. 2010. HapX-mediated adaption to iron starvation is crucial for virulence of Aspergillus fumigatus. PLOS Pathogens 6:e1001124. doi: 10.1371/journal.ppat. 1001124.

Schrettl M, Bignell E, Kragl C, Sabiha Y, Loss O, Eisendle M, Wallner A, Arst HN Jr, Haynes K, Haas H. 2007. Distinct roles for intra- and extracellular siderophores during Aspergillus fumigatus infection. PLOS Pathogens 3: 1195-1207. doi: 10.1371/journal.ppat.0030128.

Schrettl M, Kim HS, Eisendle M, Kragl C, Nierman WC, Heinekamp T, Werner ER, Jacobsen I, Illmer P, Yi H, Brakhage AA, Haas H. 2008. SreA-mediated iron regulation in Aspergillus fumigatus. Molecular Microbiology 70: 27-43. doi: 10.1111/j.1365-2958.2008.06376.x.

Slesiona S, Gressler M, Mihlan M, Zaehle C, Schaller M, Barz D, Hube B, Jacobsen ID, Brock M. 2012a. Persistence versus escape: Aspergillus terreus and Aspergillus fumigatus employ different strategies during interactions with macrophages. PLOS ONE 7:e31223. doi: 10.1371/journal.pone.0031223.

Slesiona S, Ibrahim-Granet O, Olias P, Brock M, Jacobsen ID. 2012b. Murine infection models for Aspergillus terreus pulmonary aspergillosis reveal long-term persistence of conidia and liver degeneration. The Journal of Infectious Diseases 205:1268-1277. doi: 10.1093/infdis/jis193.

Starich MR, Wikström M, Schumacher S, Arst HN Jr, Gronenborn AM, Clore GM. 1998. The solution structure of the Leu22->Val mutant AREA DNA binding domain complexed with a TGATAG core element defines a role for hydrophobic packing in the determination of specificity. Journal of Molecular Biology 277:621-634. doi: 10.1006/ jmbi.1997.1626.

Steiger MG, Blumhoff ML, Mattanovich D, Sauer M. 2013. Biochemistry of microbial itaconic acid production. Frontiers in Microbiology 4:23. doi: 10.3389/fmicb.2013.00023. 
Sun Y-P, Unestam T, Lucas SD, Johanson KJ, Kenne L, Finlay R. 1999. Exudation-reabsorption in a mycorrhizal fungus, the dynamic interface for interaction with soil and soil microorganisms. Mycorrhiza 9:137-144. doi: 10. $1007 / \mathrm{s} 005720050298$.

Temme N, Oeser B, Massaroli M, Heller J, Simon A, Collado IG, Viaud M, Tudzynski P. 2012. BcAtf1, a global regulator, controls various differentiation processes and phytotoxin production in Botrytis cinerea. Molecular Plant Pathology 13:704-718. doi: 10.1111/j.1364-3703.2011.00778.x.

Theilgaard HB, Kristiansen KN, Henriksen CM, Nielsen J. 1997. Purification and characterization of delta-(L-alphaaminoadipyl)-L-cysteinyl-D-valine synthetase from Penicillium chrysogenum. The Biochemical Journal 327:185-191.

Timmerman MM, Woods JP. 1999. Ferric reduction is a potential iron acquisition mechanism for Histoplasma capsulatum. Infection and Immunity 67:6403-6408.

Todd RB, Fraser JA, Wong KH, Davis MA, Hynes MJ. 2005. Nuclear accumulation of the GATA factor AreA in response to complete nitrogen starvation by regulation of nuclear export. Eukaryotic Cell 4:1646-1653. doi: 10 1128/EC.4.10.1646-1653.2005.

Trabolsy ZBKA, Anouar EH, Zakaria NSS, Zulkeflee M, Hasan MH, Zin MM, Ahmad R, Sultan S, Weber J-FF. 2014. Antioxidant activity, NMR, X-ray, ECD and UV/vis spectra of (+)-terrein: experimental and theoretical approaches. Journal of Molecular Structure 1060:102-110. doi: 10.1016/j.molstruc.2013.12.034.

Tudzynski B, Homann V, Feng B, Marzluf GA. 1999. Isolation, characterization and disruption of the areA nitrogen regulatory gene of Gibberella fujikuroi. Molecular \& General Genetics 261:106-114. doi: 10.1007/ s004380050950.

Upadhyay RS, Rai B. 1987. Studies on antagonism between Fusarium udum Butler and root region microflora of pigeon-pea. PLSO 101:79-93.

Vargas WA, Mukherjee PK, Laughlin D, Wiest A, Moran-Diez ME, Kenerley CM. 2014. Role of gliotoxin in the symbiotic and pathogenic interactions of Trichoderma virens. Microbiology 160:2319-2330. doi: 10.1099/mic.0. 079210-0.

Wang KL, Li H, Ecker JR. 2002. Ethylene biosynthesis and signaling networks. The Plant Cell (Suppl 14) S131-S151.

Weichselbaum TE, Hagerty JC, Mark HB Jr. 1969. A reaction rate method for ammonia and blood urea nitrogen utilizing a pentacyanonitrosyloferrate catalyzed Berthelot reaction. Analytical Chemistry 41:848-850. doi: 10. 1021/ac60275a046.

Yang SF, Hoffman NE. 1984. Ethylene biosynthesis and its regulation in higher plants. Annual Review of Plant Physiology 35:155-189. doi: 10.1146/annurev.pp.35.060184.001103.

Yin W, Keller NP. 2011. Transcriptional regulatory elements in fungal secondary metabolism. The Journal of Microbiology 49:329-339. doi: 10.1007/s12275-011-1009-1.

Yu J, Chang PK, Ehrlich KC, Cary JW, Bhatnagar D, Cleveland TE, Payne GA, Linz JE, Woloshuk CP, Bennett JW. 2004. Clustered pathway genes in aflatoxin biosynthesis. Applied and Environmental Microbiology 70: 1253-1262. doi: 10.1128/AEM.70.3.1253-1262.2004.

Zaehle C, Gressler M, Shelest E, Geib E, Hertweck C, Brock M. 2014. Terrein biosynthesis in Aspergillus terreus and its impact on phytotoxicity. Chemistry \& Biology 21:719-731. doi: 10.1016/j.chembiol.2014.03.010.

Zähner H, Keller-Schierlein W, Hütter R, Hess-Leisinger K, Deér A. 1963. Product of metabolism of microorganisms. Siderophores of Aspergillaceae. Archives of Microbiology 45:119-135.

Zarnowski R, Cooper KG, Brunold LS, Calaycay J, Woods JP. 2008. Histoplasma capsulatum secreted gammaglutamyltransferase reduces iron by generating an efficient ferric reductant. Molecular Microbiology 70:352-368. doi: 10.1111/j.1365-2958.2008.06410.x. 